\title{
High Breakdown Estimation Methods for Phase I Multivariate Control Charts
}

\author{
Willis A. Jensen, Jeffrey B. Birch, and William H. Woodall \\ Department of Statistics \\ Virginia Polytechnic Institute and State University \\ Blacksburg, VA 24061-0439 \\ (wajensen@vt.edu, jbbirch@vt.edu, bwoodall@vt.edu)
}

\begin{abstract}
The goal of Phase I monitoring of multivariate data is to identify multivariate outliers and step changes so that the estimated control limits are sufficiently accurate for Phase II monitoring. High breakdown estimation methods based on the minimum volume ellipsoid (MVE) or the minimum covariance determinant (MCD) are well suited to detecting multivariate outliers in data. However, they are difficult to implement in practice due to the extensive computation required to obtain the estimates. Based on previous studies, it is not clear which of these two estimation methods is best for control chart applications. The comprehensive simulation study here gives guidance for when to use which estimator, and control limits are provided. High breakdown estimation methods such as MCD and MVE, can be applied to a wide variety of multivariate quality control data.
\end{abstract}

KEY WORDS: Asymptotic Properties; Breakdown Point; Minimum Covariance Determinant; Minimum Volume Ellipsoid; Multivariate Outliers; Multivariate Statistical Process Control; Robust Estimation. 


\section{Introduction}

The frequency of multivariate statistical process control (SPC) applications has increased in recent years as data collection systems have become more sophisticated. Phase I of the monitoring scheme consists of determining whether or not historical data indicates a stable (or in-control) process. Phase II consists of monitoring future observations and using control limits calculated from Phase I to determine if the process continues to be in-control. In Phase I historical data, trends, step changes, outliers and other unusual data points can have an adverse effect on the resulting Phase II control limits. So it becomes very important to discover these unusual data points prior to calculating the control limits. Control limits based on data coming from unstable (or out-of-control) processes will be inaccurate and reduce the effectiveness of the Phase II scheme.

Classical estimation methods will not yield appropriate control limits if there are unusual data points in the Phase I data. Robust estimation methods have a distinct advantage over classical methods in that they are not unduly influenced by unusual data points. Consequently, they are much more effective in detecting any unusual points and ensuring that the control limits are reasonable. The term robustness refers to methods that are insensitive to departures from well behaved, independent, normally distributed data. Here the focus is on robustness to outliers which are more likely than not to be present in data.

Some robust estimation methods are well suited for detecting multivariate outliers or clusters of multivariate outliers because of their high breakdown points. The general idea of the breakdown point is "the smallest proportion of the observations which can render an estimator meaningless" (Hampel et al. (1986), Rousseeuw and Leroy (1987)). In other words, the breakdown point refers to the amount of "bad" data that can be 
present before the estimator no longer is accurate for the "good" data. The "good" data simply refers to the data that is in the majority and the "bad" data refers to the data in the minority. It is desirable to accurately determine which data is bad (if any).

Robust estimation methods for univariate quality control data (such as those based on a median or trimmed mean) are more straightforward and have received more attention in past research (See for example, Rocke (1989), Rocke (1992), Tatum (1997), Davis and Adams (2005)). Robust methods for multivariate data are not as straightforward nor as easily implemented. Robust estimation methods have been widely used in a regression context but they have only recently been introduced to multivariate quality control applications. Because of the differences that can result from competing methods, the choice of which robust estimator to use has not been made clear from previous studies (Wisnowski, Simpson, and Montgomery (2002), Vargas (2003)).

To evaluate the performance of competing methods for Phase I applications the probability of a signal is the preferred measure. When the data comes from an incontrol process then the probability of a signal should be close to a specified nominal value. When data comes from an out-of-control process then the probability of a signal should be large to ensure that the out-of-control points are not included in the calculation of the control limits for Phase II.

In this paper we give a brief overview of various high breakdown estimation methods based on the MVE and the MCD for multivariate Phase I applications. A comprehensive study allows us to determine the conditions under which each is preferred. We also give control limit values for practical use.

\section{Multivariate Outliers}

When working with p-dimensional multivariate normal data both the location and dispersion are of interest. The location is described by a mean vector which represents a 
point in the multidimensional space and the dispersion (or scatter or shape) is described by a variance-covariance matrix. Outliers in multivariate data are more difficult to detect than outliers in univariate data. One reason for this is because simple graphical methods that can be used to detect univariate outliers are often not possible in higher dimensions. Another reason is because there are many more ways that the multivariate data can come from an out-of-control process. For example, there could be outliers due to changes of location in random directions for each outlier, there could be a cluster of outliers due to a location shift in a particular direction, there could be multiple clusters of outliers in different directions, there could be points with the same location as the good data but with more variability, or the outliers can be due to a shift in some of the elements of the location vector but not all of them. The term "masking effect" has been coined to describe the situation where multiple outliers are present and inflate the estimates in such a way that they mask each other and escape detection. See Rocke and Woodruff (1993) for a discussion of various types of outliers.

Rocke and Woodruff (1996) stated that the the most difficult type of multivariate outliers to detect are those that have the same variance-covariance matrix as the good data. These difficult to detect outliers are referred to as "shift outliers" because the center of the outlying points has been shifted by some amount from the center of the good data. The categorization of shift outliers includes individual points as well as clusters of points. If shift outliers can be detected by the robust estimation method, then the method will likely work well for other kinds of outliers, hence the focus on shift outliers here.

\section{Properties of Estimators}

There are four major measures or properties that can be used to determine the usefulness of a multivariate estimator. The first, the breakdown point, has many different 
definitions, but the definition used here is the finite sample replacement breakdown point as defined by Donoho and Huber (1983). This value, $\pi$, is the smallest fraction of arbitrarily large bad data points that can be present before the estimator is impacted. As the sample size increases, $\pi$ will often converge to an asymptotic breakdown point. The asymptotic breakdown point is often used to compare different estimators.

Classical estimation methods have low breakdown points while the high breakdown estimators considered here have breakdown points that approach 50\%, the maximum possible value. The higher the breakdown point, the more resistant the estimator is to bad data. In other words, the less susceptible it is to the "masking effect".

The second property to consider is that of affine equivariance. Changing the measurement scale should not impact the properties of the estimator. Lopuhaä and Rousseeuw(1991) showed that the maximum possible asymptotic breakdown point for an affine equivariant estimator is $50 \%$. The estimators of location and dispersion that are considered in our paper are all affine equivariant (Rousseeuw and Leroy (1987)). For an example of non-affine equivariant estimators, see Maronna and Zamar (2002) who found that alternative estimators can be found by relaxing the restriction of affine equivariance.

The third property is the statistical efficiency of the estimator. This concerns how well it makes use of all the good data available. For the univariate case it is well known that while the median is very robust, it is also very inefficient when compared to the mean. There often has to be some tradeoff between increasing the breakdown point and the decreasing efficiency.

Finally, it should be possible to calculate the estimators with a reasonable amount of computing power in a reasonable amount of time. It should not always be expected that a reasonable time to compute the estimators be only a few seconds. It is good to 
spend the necessary time to get good estimators that give accurate information in the spirit of the following statement: "Statistical analysis is generally just a small part of the effort and cost of any data gathering and analysis . . . we consider it clearly far better to use an analysis that takes 10 hours but finds all the outliers than one that takes 10 seconds yet misses most outliers" (Hawkins and Olive (2002, p. 146)).

\section{High Breakdown Estimation for Multivariate SPC Data}

Robust estimation methods can be used in two different approaches. The first approach is to use the robust estimators in place of classical estimators. This has been the primary focus of a large amount of research dedicated to robust estimation procedures and is most useful in a regression context where the data does not necessarily have a given time order. Here the goal is to identify, for descriptive and predictive purposes, a good model that has not been unduly influenced by outliers. This approach has a higher priority on efficiency.

The second approach is to use the robust estimators to identify and remove outliers and then use classical estimators on the remaining "good" data points. Phase I quality control applications (both univariate and multivariate) have predominantly utilized this second approach. This second approach seems to be a reasonable trade off between the good efficiency of the classical estimates and the high breakdown point of resistant methods. Under this framework robust methods that are efficient are not as useful if they have lower breakdown points.

When using the second approach, the computability and breakdown point of the estimator become more important. As a consequence, statistical efficiency is not as crucial because the resistant estimators will eventually be replaced by classical estimators. Therefore estimators based on the minimum volume ellipsoid (MVE) and the 
minimum covariance determinant (MCD) are considered here. Algorithms for computing them are more plentiful, they are affine equivariant, and most importantly, they have high breakdown points. They have lower statistical efficiency because they only use slightly more than half of the available points, but this is of minor concern in Phase I analysis, especially when the Phase I data set is sufficiently large. The main concern in our Phase I setting is to provide protection against outliers.

There is a wide variety of robust estimation methods that are not considered here for multivariate data. For example, methods based on M-estimation have been widely used in a regression context. M-estimation seeks to appropriately down weight outliers in order to minimize their impact. As such, they are more efficient than the high breakdown methods considered here, but they have lower breakdown points that get even worse as the number of dimensions increases. Other methods include S-estimation, the projection methods of Stahel-Donoho (Rousseeuw and Leroy (1987, 7.1.c)), and the sequential point addition type methods of Hadi $(1992,1994)$ and Atkinson (1993). These other methods are usually applied to regression problems.

It is assumed that the Phase I historical data set consists of $m$ time ordered vectors that are independent of each other. Each vector is of dimension $p$, so $\mathbf{x}_{i}$ is a vector containing the $p$ measurements for the $i^{\text {th }}$ time period. When the process is in-control then each $\mathbf{x}_{i}$ is assumed to come from a multivariate normal distribution, that is, $\mathbf{x}_{i}$ $\sim \operatorname{MVN}(\boldsymbol{\mu}, \boldsymbol{\Sigma})$ where $\boldsymbol{\mu}$ is the population mean vector that determines the location and $\boldsymbol{\Sigma}$ is a $p$ by $p$ positive definite variance-covariance matrix that determines the dispersion.

Outliers can be identified by the $T^{2}$ statistic which is widely used for multivariate data analysis. The general form of the statistic is

$$
T_{i}^{2}=\left(\mathbf{x}_{i}-\boldsymbol{\mu}\right)^{\prime} \boldsymbol{\Sigma}^{-1}\left(\mathbf{x}_{i}-\boldsymbol{\mu}\right) \text { for } i=1,2, \ldots, m .
$$


Because $\boldsymbol{\mu}$ and $\boldsymbol{\Sigma}$ are not known, they are replaced with appropriate estimators. The classical estimators are the sample mean vector and sample variance-covariance matrix given by,

$$
\overline{\mathbf{x}}=\frac{\sum_{i=1}^{m} \mathbf{x}_{i}}{m}
$$

and

$$
\mathbf{S}_{1}=\frac{\sum_{i=1}^{m}\left(\mathbf{x}_{i}-\overline{\mathbf{x}}\right)\left(\mathbf{x}_{i}-\overline{\mathbf{x}}\right)^{\prime}}{m-1}
$$

A $T_{i}^{2}$ statistic based on these classical estimators is denoted by $T_{1, i}^{2}$. This statistic is equivalent to the squared Mahalanobis distance and has been shown to be effective in detecting a single moderately-sized multivariate outlier (See Figure 1 of Vargas (2003)). However, as shown by Sullivan and Woodall (1996) this statistic is not effective in detecting sustained step changes in the mean vector, nor is it effective in detecting multiple outliers (Vargas (2003)). This is because its breakdown point is an undesirable $1 / m$ which goes to 0 as the sample size increases. That is, a single arbitrarily large outlier can render the $T_{1, i}^{2}$ statistic ineffective.

An alternative is to base the $T_{i}^{2}$ statistic on the sample mean and the variancecovariance matrix based on the successive differences between vectors, denoted by $T_{2, i}^{2}$. If $\mathbf{v}_{i}=\mathbf{x}_{i+1}-\mathbf{x}_{i}$ is the vector of the $i^{\text {th }}$ successive difference, then an unbiased estimator of the variance-covariance matrix is

$$
\mathbf{S}_{2}=\frac{1}{2(m-1)} \sum_{i=1}^{m-1} \mathbf{v}_{i} \mathbf{v}_{i}^{\prime}
$$

This statistic is analogous to the use of the moving range to construct a Shewhart Individuals chart. Sullivan and Woodall (1996) showed that $T_{2, i}^{2}$ is effective in detecting sustained step changes in the process that occur in Phase I data. However, like $T_{1, i}^{2}, T_{2, i}^{2}$ will not be effective in detecting multiple multivariate outliers because its breakdown point is also $1 / m$, thus it will not be considered here (Vargas (2003)). 
Robust alternatives of the $T^{2}$ statistics considered here are based on either the minimum volume ellipsoid (MVE) estimator or the minimum covariance determinant (MCD) estimator These will be denoted by $T_{m v e, i}^{2}$ or $T_{m c d, i}^{2}$ respectively, and defined as:

$$
\begin{aligned}
& T_{m v e, i}^{2}=\left(\mathbf{x}_{i}-\mathbf{x}_{m v e}\right)^{\prime} \mathbf{S}_{m v e}^{-1}\left(\mathbf{x}_{i}-\mathbf{x}_{m v e}\right) \text { for } i=1,2, \ldots, m, \\
& T_{m c d, i}^{2}=\left(\mathbf{x}_{i}-\mathbf{x}_{m c d}\right)^{\prime} \mathbf{S}_{m c d}^{-1}\left(\mathbf{x}_{i}-\mathbf{x}_{m c d}\right) \text { for } i=1,2, \ldots, m .
\end{aligned}
$$

where $\mathbf{x}_{m v e}$ and $\mathbf{x}_{m c d}$ are the corresponding location estimators and $\mathbf{S}_{m v e}$ and $\mathbf{S}_{m c d}$ are the corresponding estimators of the variance-covariance matrix. In the following sections we discuss these estimators in more detail and explain how they are calculated.

\section{Minimum Volume Ellipsoid Estimator}

The minimum volume ellipsoid (MVE) estimator, first proposed by Rousseeuw (1984), has been studied extensively for non-control chart settings and frequently used in the detection of multivariate ouliers. It seeks to find the ellipsoid of minimum volume that covers a subset of at least $h$ data points. Subsets of size $h$ are called halfsets because $h$ is often chosen to be just more than half of the $m$ data points. The location estimator is the geometrical center of the ellipsoid and the estimator of the variance-covariance matrix is the matrix defining the ellipsoid itself, multiplied by an appropriate constant to ensure consistency (Rousseeuw and van Zomeren (1990), Rousseeuw and Van Zomeren (1991), and Rocke and Woodruff (1996)). Thus the MVE estimator of location and dispersion do not correspond to the sample mean vector and sample variance-covariance matrix of a particular halfset.

To achieve the highest breakdown point possible, Davies (1987) and Lopuhaä and Rousseeuw (1991) showed that the integer value of $h=(m+p+1) / 2$ should be used for

the MVE. This will achieve a breakdown value of $\frac{[(m-p+1) / 2]}{m}$ percent which converges 
to $50 \%$ as $m \rightarrow \infty$. The value of $h$ can be increased, to say, $.75 m$, if it is believed that the percentage of bad data is low. This will increase the efficiency of the the MVE estimator. However caution must be exercised because the consequences of having a value of $h$ higher than the number of good data points is more severe (contaminated estimates) than the consequences of having a value of $h$ lower than the number of good data points (loss of statistical efficiency but still giving good estimates). For this reason, $h$ is often set to achieve the highest breakdown point possible, as is the case for this paper.

Finding the MVE estimators is essentially a two-part process. One part is to find the best halfset consisting of $h$ points. Then the second part requires finding the ellipsoid of minimum volume that covers the halfset. For a given halfset there are many ellipsoids that cover it. Titterington (1975) found that the solution to this second step is equivalent to finding a D-optimal design for a design region where the points in a halfset are the design points. As a consequence, iterative algorithms to find D-optimal designs could be used to find the best covering ellipsoid for the best halfset. The first step is referred to as the "subset" problem and the second step is referred to as the "covering problem" (Agullo (1996)).

While the idea of the MVE is very intuitive, actually finding the MVE estimator can be very difficult in practice. As the sample size $(m)$ and data dimension $(p)$ increase, the required computational effort increases exponentially. For example, if $m=30$ and $p=3$, so that $h=\frac{30+3+1}{2}=17$, then there are a total of $\frac{30 !}{13 ! 17 !}=119,759,850$ halfsets that could potentially be the basis for the MVE estimator. Even when this halfset is found, it still takes additional calculations to find the best covering ellipsoid. As a consequence of the computational difficulty, Rousseeuw and Leroy (1987) proposed an approximate method to find the MVE estimators by a subsampling algorithm. 
The subsampling algorithm is very commonly used, is widely accessible, and is the basis of the MVE functions of software packages such as S-Plus and SAS. This subsampling algorithm takes a fixed number of random subsets, known as elemental subsets, each containing $p+1$ points. For each elemental subset, the sample mean vector and sample variance-covariance matrix are calculated, which determines the shape of an ellipsoid. This ellipsoid is then increased in size by multiplying by a constant until it covers at least $h$ data points. The ellipsoid with the smallest volume is then used to obtain the MVE estimates.

Rousseeuw and Leroy (1987, p. 199) recommended doing a minimum of 500 subsamples for small datasets with low dimensions. More subsamples should be used as $m$ and $p$ increase. Rousseeuw and Leroy $(1987$, p. 260) also showed that if $\epsilon$ is the true proportion of outliers in the dataset then a probabilistic argument can be used to determine the number of random subsamples $(j)$ needed to ensure with a high probability that at least one contains only good points. The approximate probability that at least one sample contains only good points is

$$
\alpha=1-\left(1-(1-\epsilon)^{p+1}\right)^{j}
$$

and (7) can be rewritten to solve for $j$ as

$$
j=\frac{\ln (1-\alpha)}{\ln \left(1-(1-\epsilon)^{p+1}\right)} .
$$

Use of (8) shows that when $p \leq 5$ and $\epsilon \leq .50$ then 500 subsamples will ensure that $\alpha$ will be greater than .999. For $p \leq 10$ and $\epsilon \leq .50$ then 10,000 subsamples will ensure that $\alpha$ will be greater than .99 .

A similar argument along these lines to determine the probability that a particular halfset will contain only good points shows that the number of halfsets that need to be considered is very large indeed. To see this, replace the value of $p+1$ (the size of 
Table 1: Number of halfsets that need to be considered to ensure that one only contains good points with probability .95 where $\mathrm{p}=3$

\begin{tabular}{|c|c|c|c|c|c|c|c|c|c|c|}
\hline & \multicolumn{10}{|c|}{$\epsilon$} \\
\hline $\mathrm{m}$ & 0.05 & 0.1 & 0.15 & 0.2 & 0.25 & 0.3 & 0.35 & 0.4 & 0.45 & 0.5 \\
\hline 20 & 4 & 10 & 20 & 43 & 94 & 215 & 526 & 1375 & 3909 & 12270 \\
30 & 6 & 17 & 46 & 132 & 398 & 1287 & 4538 & 17697 & 77684 & 392656 \\
40 & 8 & 29 & 106 & 405 & 1678 & 7661 & 39120 & 227601 & 1543560 & 12565011 \\
50 & 11 & 51 & 240 & 1238 & 7076 & 45588 & 337164 & 2926976 & 30669759 & 402080378 \\
60 & 14 & 86 & 542 & 3780 & 29821 & 271246 & 2905865 & 37641158 & 609392261 & 12866572141 \\
70 & 19 & 147 & 1224 & 11538 & 125670 & 1613895 & 25044294 & 484068397 & 12108307535 & $4.1173 \mathrm{E}+11$ \\
80 & 25 & 249 & 2759 & 35214 & 529577 & 9602521 & 215845013 & 6225159088 & $2.40586 \mathrm{E}+11$ & $1.31754 \mathrm{E}+13$ \\
90 & 32 & 423 & 6219 & 107466 & 2231636 & 57134063 & 1860266840 & 80056126192 & $4.78001 \mathrm{E}+12$ & $4.21612 \mathrm{E}+14$ \\
100 & 42 & 717 & 14018 & 327961 & 9404100 & 339942070 & 16032763795 & $1.02954 \mathrm{E}+12$ & $9.50111 \mathrm{E}+13$ & $1.34916 \mathrm{E}+16$ \\
\hline
\end{tabular}

the elemental subset) in (8) with the integer value of $h=(m+p+1) / 2$ (the size of the halfset). Table 1 below shows the number of halfsets that need to be considered when $p=3$ to have a $95 \%$ chance of getting one containing only good points. This illustrates the difficulty in finding a good MVE estimator, particularly as the sample size and the proportion of bad data increases.

In addition, it is important to recognize that while this subsampling approach is computationally easier, it is only an approximation. Even when elemental subsets with good points are used, this does not ensure that the resulting halfsets will only have good points. This is because the resulting ellipsoid that covers the halfset is proportional to the ellipsoid for the corresponding elemental subset, which is not necessarily the minimum volume ellipsoid for the halfset. An exhaustive calculation using all possible elemental subsets will still yield an approximate estimator (Cook and Hawkins (1990)). This is because the ellipsoid for the MVE estimator is not necessarily proportional to the ellipsoid for any of its elemental subsets. In fact, it is probably more likely that the MVE is not proportional to the ellipsoid for one of its elemental subsets.

While the number of possible elemental subsets is smaller than the number of 
possible halfsets, there is still the same exponential increase in number of possible elemental subsets as $m$ and $p$ increase. For the example shown earlier with $m=30$ and $p=3$ there are a total of $\frac{30 !}{4 ! 26 !}=27,405$ elemental subsets that need to be considered if an exhaustive calculation were done. The exponential increase in the number of elemental subsets needed to ensure a good approximate estimate limits the types of problems that can be analyzed using the MVE.

Finally, there are repeatability issues with this subsampling approach. If an exhaustive calculation using all possible elemental subsets is not done, then two different analyses on the same data set will likely yield different results. The difference in the results gets more severe as $m$ and $p$ get larger because for a fixed number of random elemental subsets, the proportion of subsets that can be feasibly calculated relative to the total number of available subsets gets smaller.

For an example of the repeatability issues of the subsampling method, Vargas (2003) calculated the $T_{m v e, i}^{2}$ statistics based on the MVE estimators using the subsampling algorithm for the data of Quesenberry (2001). Table 2 shows the results obtained by Vargas (Using S-PLUS), our results using the "call mve" functions of SAS for 500 subsamples, and our results using all possible subsamples, of which there are $\frac{30 !}{3 ! 27 !}=$ 4,060. Notice here that different values are obtained depending on the number of subsamples used. It is not clear what number of subsamples or what covering methods that Vargas (2003) used, but the differences in values is cause for concern.

To avoid some of the difficulties with the subsampling approach, an exact method to calculate the MVE estimators was proposed by Cook, Hawkins, and Weisberg (1993). It considers all the possible halfsets and would require an enormous amount of computation even for modest sample sizes in lower dimensions. Once the best halfset is found the "covering" solution is found using the approach of Titterinton (1975). To speed up 


\begin{tabular}{|c|c|c|c|}
\hline observations & MVE(500 subsamples in SAS) & MVE(all subsamples in SAS ) & MVE(Vargas (2003)) \\
\hline 1 & 0.860 & 0.921 & 0.835 \\
\hline 2 & 30.803 & 24.960 & 25.770 \\
\hline 3 & 0.484 & 0.353 & 0.432 \\
\hline 4 & 2.700 & 2.614 & 2.398 \\
\hline 5 & 1.420 & 1.506 & 1.434 \\
\hline 6 & 0.274 & 0.313 & 0.227 \\
\hline 7 & 1.282 & 1.292 & 1.143 \\
\hline 8 & 1.126 & 0.928 & 1.039 \\
\hline 9 & 0.066 & 0.094 & 0.064 \\
\hline 10 & 1.064 & 1.034 & 0.867 \\
\hline 11 & 0.970 & 0.768 & 0.878 \\
\hline 12 & 1.332 & 1.033 & 1.175 \\
\hline 13 & 0.560 & 0.585 & 0.467 \\
\hline 14 & 6.815 & 6.101 & 5.712 \\
\hline 15 & 0.220 & 0.121 & 0.183 \\
\hline 16 & 5.212 & 4.949 & 5.117 \\
\hline 17 & 2.194 & 2.303 & 2.268 \\
\hline 18 & 3.014 & 3.151 & 3.060 \\
\hline 19 & 1.865 & 1.868 & 1.702 \\
\hline 20 & 6.770 & 6.569 & 6.736 \\
\hline 21 & 1.818 & 1.899 & 1.885 \\
\hline 22 & 7.896 & 5.952 & 6.385 \\
\hline 23 & 0.367 & 0.390 & 0.380 \\
\hline 24 & 1.119 & 1.146 & 1.012 \\
\hline 25 & 1.580 & 1.631 & 1.637 \\
\hline 26 & 0.477 & 0.440 & 0.476 \\
\hline 27 & 0.604 & 0.509 & 0.576 \\
\hline 28 & 5.648 & 4.265 & 4.622 \\
\hline 29 & 3.977 & 3.044 & 3.329 \\
\hline 30 & 0.182 & 0.218 & 0.181 \\
\hline
\end{tabular}

Table 2: Comparison of $T_{m v e, i}^{2}$ obtained via MVE subsampling algorithm for Quesenberry (2001) data 
the algorithm they proposed a modification based on the fact that the ellipsoid cannot decrease in volume with each successive iteration. The volume is measured by the determinant so in the algorithm if a subset of points yields a value for the determinant larger than the current best value, then the halfset is not evaluated any further. This modification allows the calculation of the exact MVE without the explicit calculation of the minimum covering for every halfset. This speeds up the algorithm considerably, as a great majority of halfsets do not require explicit calculation. Cook, Hawkins, and Weisberg (1993) found that for typical datasets fewer than $1 \%$ of the possible halfsets require explicit evaluation. However, even with this speedup of the algorithm, this exact method is still only feasible for small datasets where $m \leq 30$ and $p \leq 5$ (Cook, Hawkins, and Weisberg (19993)).

Agullo (1996) proposed an exact method to calculate the MVE estimators based on a more computationally efficient branch and bound method. Similar to the modification proposed by Cook, Hawkins, and Weisberg (1993) to speed up their algorithm, the branch and bound method utilizes the fact that the volume of a subset of points cannot decrease as additional points are added. In other words, the volume is monotonically non-decreasing as points are added to the subset. For example, consider the situation with $p=2, m=30$, and $h=16$. During the search if a subset of 8 points is found to have a higher volume (as measured by a determinant) than the best halfset found to that point, then no further halfsets containing those 8 points need to be considered. This reduces substantially the number of halfsets for which a determinant is calculated. Once the best halfset is found, Agullo (1996) recommended using an algorithm by Atwood (1973) that is faster than the approach of Titterington (1975) to solve the "covering problem". The branch and bound algorithm can be sped up by ordering the data prior to beginning the search. As a result, the branch and bound 
method is computationally feasible for datasets where $m \leq 100$ and $p \leq 5$.

Other computationally feasible methods to find an approximate MVE have been proposed. For example, Hawkins (1993) proposed a feasible solution algorithm (FSA). This algorithm considers a randomly selected halfset (called a random start) and then makes use of swapping techniques to find a better halfset for which its covering ellipsoid is found. Then the procedure is repeated for many randomly selected halfsets, each of which converges to a local feasible solution. The MVE estimators are based on the minimum of the local solutions. If enough randomly selected halfsets are used, this algorithm will eventually yield an exact solution, but this will not be guaranteed for a finite number of halfsets. If we denote by $\theta$ the proportion of initial halfsets that will yield the best halfset, then the probability of finding the exact result, $\operatorname{Pr}($ exact), is $1-(1-\theta)^{N}$ where $N$ is the number of random starts. This expression can be use to determine the number of random starts that is needed to achieve a certain probability of getting the exact results. Hawkins (1993) showed that for many common datasets previously studied in the literature (with $m \leq 50$ and $p \leq 5$ ) that the $N$ required to achieve a high probability of success is often less than 100 (See also Hawkins (1994)) so the computation time is substantially smaller than those of Cook, Hawkins, and Weisberg (1993) and Agullo (1996).

Croux and Haesbroeck $(1997,2002)$ showed how the efficiency of the subsampling approach can be improved for the MVE. Instead of just picking the optimal elemental subset that gives the minimum volume, they first computed the ordered minimum volumes and then averaged some of the smallest ones. These estimators still retain consistency, affine equivariance, and have the highest possible breakdown point. However, it is still an approximate method and if an exhaustive calculation is not done, this averaged approach still has the repeatability problem. This approach will not be con- 
sidered here because of the additional computation complexity with only a relatively small gain in efficiency which is of minor importance.

Methods to find the MVE based on a heuristic search algorithms were proposed by Woodruff and Rocke (1993). These search algorithms reduce the amount of computing time needed to solve the "subset" problem and include genetic algorithms, simulated annealing, and their corresponding enhanced versions. While they were shown to be much more computationally efficient than the subsampling method and give good results, they are not considered here because the algorithms are not easily accessible.

\section{Minimum Covariance Determinant Estimator}

An alternative high breakdown estimation procedure to the MVE is an estimator based on the minimum covariance determinant (MCD), which was first proposed by Rousseeuw (1984). It is obtained by finding the halfset that gives the minimum value of the determinant of the variance-covariance matrix. The resulting estimator of location is the sample mean vector of the points that are in the halfset and the estimator of the dispersion is the sample variance-covariance matrix of the points multiplied by an appropriate constant to ensure consistency just as was done for the MVE. Thus in contrast to the MVE, the MCD estimators correspond to $\overline{\mathbf{x}}$ and $\mathbf{S}_{1}$ of a specific halfset. Because the MCD estimators are simple to calculate once the best halfset is found, it can be easier to compute than for the MVE as it does not require a solution to the "covering problem".

The MCD estimators are intuitively appealing because a small value of the determinant corresponds to near linear dependencies of the data in the $p$-dimensional space. That is because a small determinant corresponds to a small eigenvalue which suggests a near linear dependency that suggests that there is a group of points that are similar to each other. 
Like the MVE, the MCD estimators have the same maximum breakdown point which is achieved when $h$ is the integer value of $(m+p+1) / 2$. In addition, the MCD estimators can be very computationally difficult to obtain because of the exponential increase in the number of potential halfsets that need to be considered. As a result, the approximate methods and algorithms to obtain MVE estimates can also be used to obtain the MCD estimates. For example, MCD estimates can be computed via the method of Cook, Hawkins, and Weiser (1993). The branch and bound method of Agullo (1996) can also be used, as shown in Agullo (2001). The subsampling approach of Rousseeuw and Leroy (1987) can be used to get an approximate MCD estimates which would have the same repeatability issues as the approximate MVE obtained via subsampling. The FSA of Hawkins (1993) can be implemented for the MCD, as shown by Hawkins (1994). An improved version of the FSA for the MCD was proposed by Hawkins and Olive (1999).

\section{Hybrid Algorithms}

Other high breakdown estimation methods for detecting multivariate outliers are hybrid algorithms that combine various components of earlier methods with modifications. Two notable ones are the hybrid algorithm of Rocke and Woodruff (1996) and the FAST-MCD algorithm of Rousseeuw and Van Driessen (1999).

The hybrid algorithm of Rocke and Woodruff (1996) is a combination of the data partitioning methods of Woodruff and Rocke (1994), the FSA algorithm involving the MCD from Hawkins (1994), a sequential point addition algorithm, and M-estimation. This hybrid algorithm is very effective in detecting a larger percentage of outliers. A more complete explanation of the algorithm and the justification for its various components can be found in Rocke and Woodruff (1997). 
Rousseeuw and Van Driessen (1999) proposed a hybrid algorithm which they called the FAST-MCD that is based on an iterative scheme and the MCD estimators. The algorithm can be described as follows:

1. Start with a fixed number, $(A)$, of random elemental subsets and use them to construct corresponding halfsets.

2. Carry out two concentration steps (C-step) on the $A$ halfsets and select a small number of "best" ones.

3. For the "best" halfsets, carry out C-steps until convergence and the FAST-MCD estimators are based on the halfset with the lowest determinant of the covariance matrix.

The C-steps are based on the fact that for any given halfset and its estimates of location and dispersion, a better (or at least equivalent) solution can be found by reordering the observations of the full dataset according to their Mahalanobis distances. A new and improved halfset of the reordered points is found by selecting from the full dataset those with the smallest Mahalanobis distances. The new halfset will have a smaller determinant of the variance-covariance matrix than the determinant of the original halfset. So each C-step yields a halfset that is more concentrated than the previous halfset. If enough C-steps are done on enough halfsets, convergence to the exact MCD estimator results. Because not all halfsets are considered, the FAST-MCD will be an approximate method unless a large enough number of initial halfsets are considered.

The FAST-MCD method is able to handle large data sets within a reasonable amount of time. In fact, Rousseeuw and Van Driessen (1999) successfully analyzed a data set with $m=132,402$ and $p=27$, which is certainly beyond the capabilities of all 
the algorithms discussed earlier. For smaller datasets that they analyzed (all with $m \leq$ 75 and $p \leq 5$ ), the FAST-MCD algorithm resulted in estimates that were equivalent to the exact MCD estimates. This means that the number of halfsets considered was large enough to achieve convergence to the exact MCD estimates. It remains to be seen how large $m$ and $p$ can be and still obtain the exact result with a high probability. The control charts that are considered here generally use smaller values of $m$ and $p$ suggesting that the FAST-MCD for practical purposes is likely to give the exact result.

Because it is not drawing random samples of points, the FAST-MCD algorithm does not have the repeatability issues that are present in the subsampling algorithm. Thus the FAST-MCD serves as a better algorithm to obtain the MCD estimator than the subsampling algorithm for the MVE estimator.

\section{Asymptotic Properties}

The MCD and MVE estimators have been used historically as a starting point for other robust estimation procedures, such as M-estimation. As such, it has not been as important that the MCD and MVE estimators be exact. However, in Phase I quality control applications, the MCD and MVE are used directly to determine multivariate outliers and thus it becomes more important that they be sufficiently accurate. It is also important to have some understanding of the distributions of the MCD and MVE estimators in order to be able to obtain appropriate control limits for the $T_{m v e, i}^{2}$ and $T_{m c d, i}^{2}$ statistics. The distributions of the exact MCD and MVE estimators of location and dispersion are not known in closed form. So when quantiles are needed from the distributions to calculate control limits, they have been found via simulation (See for example, Vargas (2003) or Williams, Woodall, and Birch 2005)).

However, the asymptotic distributions of the MVE and MCD estimators can be derived. Davies $(1987,1992)$ showed that the exact MVE estimators of location and 
dispersion are consistent for $\boldsymbol{\mu}$ and $\boldsymbol{\Sigma}$ given that the $\mathbf{x}_{i}$ are independently and identically distributed with a common distribution. Butler, Davies, and Jhun (1993) showed the corresponding result for the exact MCD estimators of location and dispersion. However, the MCD estimators converge to its population counterparts at a rate of $n^{-1 / 2}$ while the MVE estimators converge at a slower rate of $n^{-1 / 3}$, thus the MCD estimators are more efficient. In addition, the distribution of the MCD estimator of location converges to a normal distribution, which is not necessarily the case for the MVE estimator of location. Thus, the asymptotic properties of the MCD estimators are superior to those of the MVE estimators. An intuitive reason for the superior convergence properties of the MCD can be found by noting that as $\epsilon \rightarrow 0$ the location MVE estimator converges to the center of the ellipsoid covering all the data while the location MCD estimator converges to the mean vector of all the points.

The asymptotic distributions of the $T_{m v e, i}^{2}$ and $T_{m c d, i}^{2}$ statistics follow directly from the consistency of the MVE and MCD estimators, as seen in the following theorems.

Theorem 1. As $m \rightarrow \infty$, the distribution of $T_{m v e, i}^{2}$ converges in distribution to a $\chi_{p}^{2}$ distribution for $i=1, \ldots, m$.

Proof. The assumption of multivariate normality satisfies the conditions of Theorem 3 of Davies (1987), therefore the MVE estimators are consistent, i.e., they converge in probability to their parameter values, so we write $\mathbf{x}_{m v e} \stackrel{p}{\rightarrow} \boldsymbol{\mu}$ and $\mathbf{S}_{m v e}^{-1} \stackrel{p}{\rightarrow} \boldsymbol{\Sigma}$ as $m \rightarrow \infty$. Thus we then have

$$
T_{m v e, i}^{2}=\left(\mathbf{x}_{i}-\mathbf{x}_{m v e}\right)^{\prime} \mathbf{S}_{m v e}^{-1}\left(\mathbf{x}_{i}-\mathbf{x}_{m v e}\right) \stackrel{p}{\rightarrow}\left(\mathbf{x}_{i}-\boldsymbol{\mu}\right)^{\prime} \boldsymbol{\Sigma}^{-1}\left(\mathbf{x}_{i}-\boldsymbol{\mu}\right) \sim \chi_{p}^{2}
$$

Theorem 2. As $m \rightarrow \infty$, the distribution of $T_{m c d, i}^{2}$ converges in distribution to a $\chi_{p}^{2}$ distribution for $i=1, \ldots, m$. 
Proof. Same as the proof of Theorem 1 but replacing Theorem 3 of Davies (1987) with Theorem 3 of Butler, Davies, and Jhun (1993) to show the consistency of the MCD estimators.

It should be noted that because the subsampling algorithm to obtain the MVE estimators and the FAST-MCD algorithm are approximations, their asymptotic distributions are not necessarily $\chi_{p}^{2}$. If it were computationally feasible to compute exactly the MVE and MCD estimators, then the control limits could be easily approximated using the quantiles of the $\chi_{p}^{2}$ distribution when the Phase I sample size is large. It should also be noted that as the proportion of bad points, $\epsilon$, goes to 0 , the $T_{m c d, i}^{2}$ statistic converges to the $T_{1, i}^{2}$ statistic which has a $\chi_{p}^{2}$ distribution.

\section{Control Limits}

Because the distribution of the $T^{2}$ statistic based on the MVE and MCD estimators are only known asymptotically, implementation of the Phase I control chart requires control limits to be generated via simulation. Appendix A contains the control limits for $T^{2}$ statistic based on the MVE estimators obtained via the subsampling method and for the MCD estimators obtained via the FAST-MCD method. To obtain the simulated control limits, 200,000 data sets were generated for each combination of $m$ and $p$ with a zero mean vector and the identity covariance matrix. Due to the invariance of the $T_{m v e, i}^{2}$ and $T_{m c d, i}^{2}$ statistics, these limits will be applicable for any values of $\boldsymbol{\mu}$ and $\boldsymbol{\Sigma}$. $T^{2}$ statistics for each observation in the data set were calculated and the maximum value attained for each data set was recorded. The $95^{\text {th }}$ percentile of this generated empirical distribution is the simulated control limit. As will be seen shortly, $T_{m v e, i}^{2}$ and $T_{m c d, i}^{2}$ will be preferred for different situations, thus the control limits are only provided for the situations where the particular estimator is preferred. 
The control limits are dependent on the integer value of $h$ used and are not monotonic functions of $m$. For example, consider Figure 1, which shows the scatterplot of the control limit of $T_{m c d, i}^{2}$ vs. $m$ for $p=3$. The jigsaw pattern here is also present when using $T_{m v e, i}^{2}$ and is due to the fact that the integer value of $h$ is the same for successive values of $m$.

\section{Control limits for MCD estimator with $p=3$}

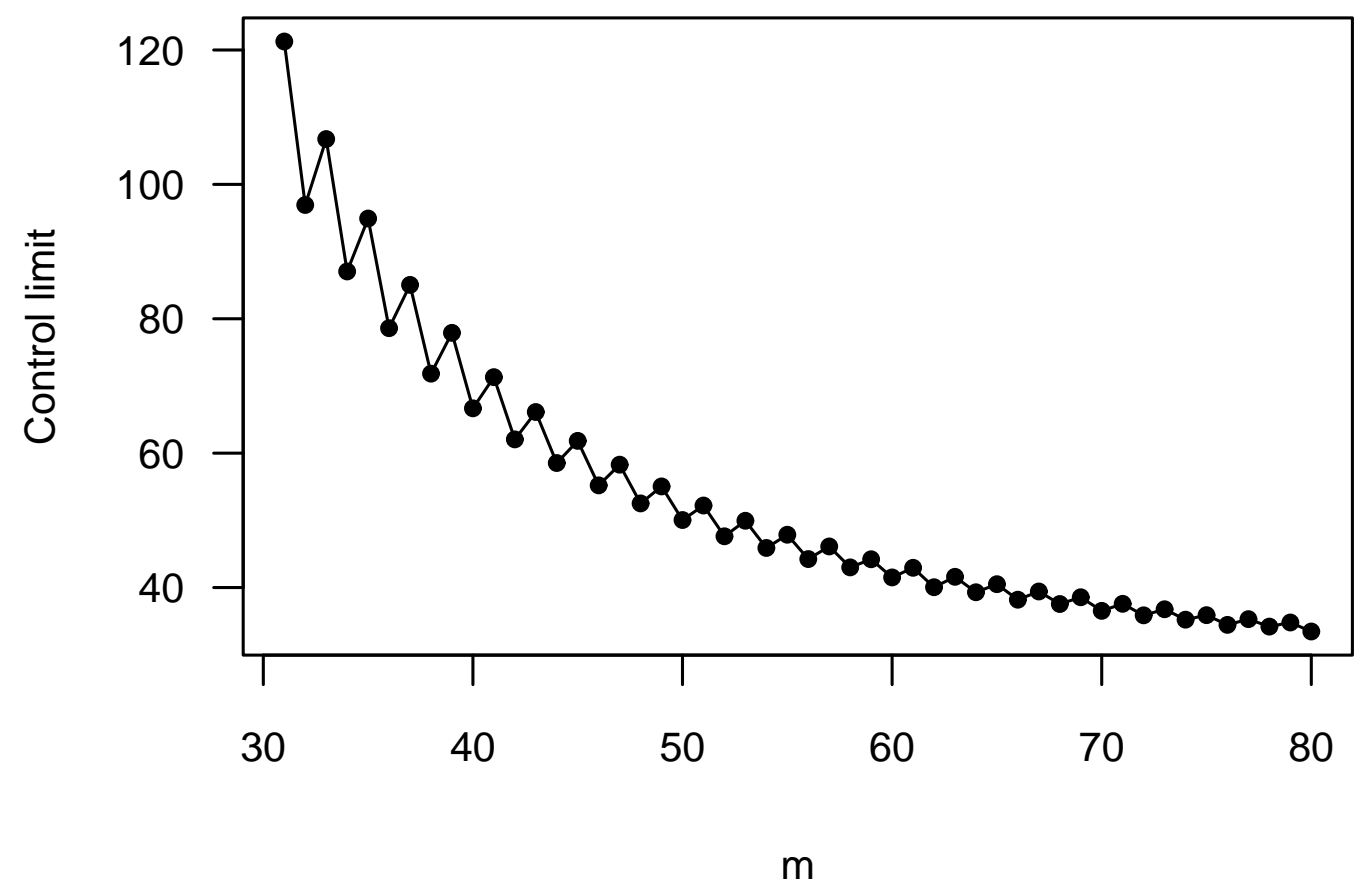

Figure 1: Scatterplot of simulated control limits for the MCD estimator versus the size of the data set, $\mathrm{m}$.

It should be noted that the control limits in Appendix A are only appropriate for the particular algorithm used. That is, the limits for $T_{m c d, i}^{2}$ are appropriate when the FAST-MCD algorithm is used and the limits for $T_{m v e, i}^{2}$ are appropriate when the 
MVE estimator with subsampling is used. Here the number of subsamples for the MVE estimator is the default number based on the SAS MVE algorithm. A difference in the algorithm changes the variability of the results from that algorithm and thus the generated control limits would vary. Because the resulting estimates can vary depending on which robust estimation algorithm is used, it is helpful to think of the "algorithm as the estimator" as discussed by Woodruff and Rocke (1994, p. 889). The variability in the estimator can be due in part to the variability in the algorithm used to obtain it. Also these control limits all use the integer value of $h=(m+p+1) / 2$, which gives the maximum possible breakdown point. Using a different value of $h$ will change the appropriate control limit.

\section{Simulation Study}

With the generated control limits, we made some comparisons of the high breakdown estimators. Vargas (2003) did a simulation study to compare $T_{1, i}^{2}, T_{2, i}^{2}, T_{m v e, i}^{2}$ obtained via subsampling, and $T_{m c d, i}^{2}$ obtained via the FAST-MCD. He concluded that the MVE gave the best performance in terms of probability of a signal when outliers are present. However, his comparisons between the MVE obtained by subsampling and the FAST-MCD only covered the case for $p=2$ and $m=30$. Wisnowski, Simpson and Montgomery (2002) did a performance study via simulation to compare various types of robust estimation procedures. They compared a sequential point addition algorithm of Hadi (1992, 1994), M-estimation, the approximate MVE calculated by the subsampling method, the FAST-MCD and the hybrid algorithm of Rocke and Woodruff (1996). However, their comparisons of the MVE obtained via subsampling and the FAST-MCD for large shift outliers only used $m=40,60, p=2,6$, and $\epsilon=10 \%, 20 \%$. Wisnowski, Simpson, and Montgomery (2002) concluded that the hybrid algorithm 
performed best and that the FAST-MCD was slightly better than the MVE based on simulation runs involved 1000 datasets. They also considered various other outlier situations not considered here such as: Outliers scattered in random directions, clusters of outliers in all $p$ variables, clusters of outliers in one of $p$ variables, clusters of outliers in some of the $p$ variables, and multiple clusters in close proximity.

We performed a similar study to those of Wisnowski, Simpson, and Montgomery (2002) and Vargas (2003) to compare the MVE subsampling and FAST-MCD algorithms. Our study compares more combinations of $p, m$, and $k$. For a particular combination of $p, m$, and $k$, a number of datasets were generated. Of the $m$ observations, $k$ of them are random data points generated from the out-of-control distribution, and the other $n-k$ observations were generated from the in-control distribution.

The in-control distribution is a multivariate normal where it can be assumed that $\boldsymbol{\mu}=\mathbf{0}$ and $\boldsymbol{\Sigma}=\mathbf{I}$ without loss of generality. The out-of-control distribution is a multivariate normal with the same variance-covariance matrix but where the mean vector has been shifted by some amount. This amount depends on a value of the non-centrality parameter, given by

$$
\left(\boldsymbol{\mu}_{1}-\boldsymbol{\mu}\right)^{\prime} \boldsymbol{\Sigma}^{-1}\left(\boldsymbol{\mu}_{1}-\boldsymbol{\mu}\right)
$$

where $\boldsymbol{\mu}_{\mathbf{1}}$ is the amount that the mean vector has shifted. The larger the value of the non-centrality parameter, the more extreme the outliers are. The proportion of datasets that had a least one $T^{2}$ statistic greater than the control limit was calculated and this proportion becomes the estimated probability of a signal. $T_{1, i}^{2}$ was included in our study as a reference statistics because of its common usage.

Appendix B shows the probability of a signal for different values of the noncentrality parameter and for some of the values of $m$ and $k$ considered in our study. For $p=2,3$, and 5 a total of 100,000 datasets of size $m$ were generated for each com- 
bination of $m, k$, and the non-centrality parameter. For $p=7$ and 10, 50, 000 datasets were generated for each combination. The results are shown in figures B.1-B.5 for $p=2,3,5,7$, and 10 respectively. As expected, when the value of the non-centrality parameter is small, the probability of a signal is close to .05 which is what would be expected for an in-control process. As the value of the non-centrality parameter increases the probability of a signal will increase. If not, then this indicates that the estimator has broken down and is not capability of detecting the outliers. In general, for small values of $m, T_{m v e, i}^{2}$ performs best, unless the number of outliers is large. As $m$ increases, $T_{m c d, i}^{2}$ is more likely to be superior. The actual breakdown point of $T_{m v e, i}^{2}$ is smaller than that of $T_{m c d, i}^{2}$ although in theory they should have similar breakdown points. It is clear that $T_{1, i}^{2}$ possesses little ability to detect multiple outliers. As $p$ increases for a fixed value of $m$, the breakdown points of $T_{m v e, i}^{2}$ and $T_{m c d, i}^{2}$ get smaller. This suggests that the larger $p$ is, the larger $m$ will need to be in order to minimize the impact of outliers. In general, there was always one estimator that was found to be superior across all the values of the non-centrality parameter as long as the proportion of outliers was not so big as to cause the estimators to break down. This greatly simplifies the conclusions that can be made about when the MVE or MCD estimator is preferred.

Figures 2-6 summarizes the results from Appendix B by showing which of the three estimators (Standard, MVE, MCD) is preferred for the various combinations of $m$, $p$, and $\epsilon$. Based on Figures 2-6 some broad recommendations can made made. The standard estimator should be used if at most one outlier is expected. When $m \leq 50$ the MVE will be the best estimator unless the percentage of outliers is greater than 25 or $30 \%$. When $m>50$, the MCD is preferred as long as the percentage of outliers is less than $40 \%$. As $p$ increases, then the percentage of outliers that can be detected by 
the MVE estimator will decrease until it is only $10 \%$ for $p=10$. It is true for both the MVE and MCD that the higher $p$ is, then the number of outliers that can be detected decreases. Thus for Phase I applications where the number of outliers is unknown $T_{m v e, i}^{2}$ should only be used for smaller sample sizes for which it is also computationally feasible. $T_{m c d, i}^{2}$ should be used for larger sample sizes or when it is believed that there is a large number of outliers. The more variables that are monitored $(p)$ the larger the sample size that will be needed to ensure that the estimator does not breakdown and lose its ability to detect any outliers.

\section{Open questions and future research}

There are still some unanswered questions related to high breakdown estimation methods for multivariate control charts. For example, because the asymptotic distri-

bution of the $T_{m c d, i}^{2}$ and $T_{m v e, i}^{2}$ is $\chi_{p}^{2}$, it may be useful to study the use of approximate control limits which are much simpler to obtain than those obtained via simulation. This type of study was performed for $T_{2, i}^{2}$ in Williams et al. (2005) to compare the probability of signal using the simulated control limits versus the asymptotic $\chi_{p}^{2}$ limit. We believe that it is likely that large sample sizes are needed for the $\chi_{p}^{2}$ approximation to be sufficiently accurate.

We have only considered here high breakdown estimation methods that are robust in the sense that they are resistant to outliers. It is not clear if these high breakdown estimation methods are robust to other departures from the specified assumptions. For example, it is not clear if the superiority of high breakdown methods is maintain when the data no longer follows a multivariate normal distribution.

Traditional methods of quality control for Phase I use the second approach, that of estimate, delete, re-estimate. It would possible to utilize the first approach and simply 


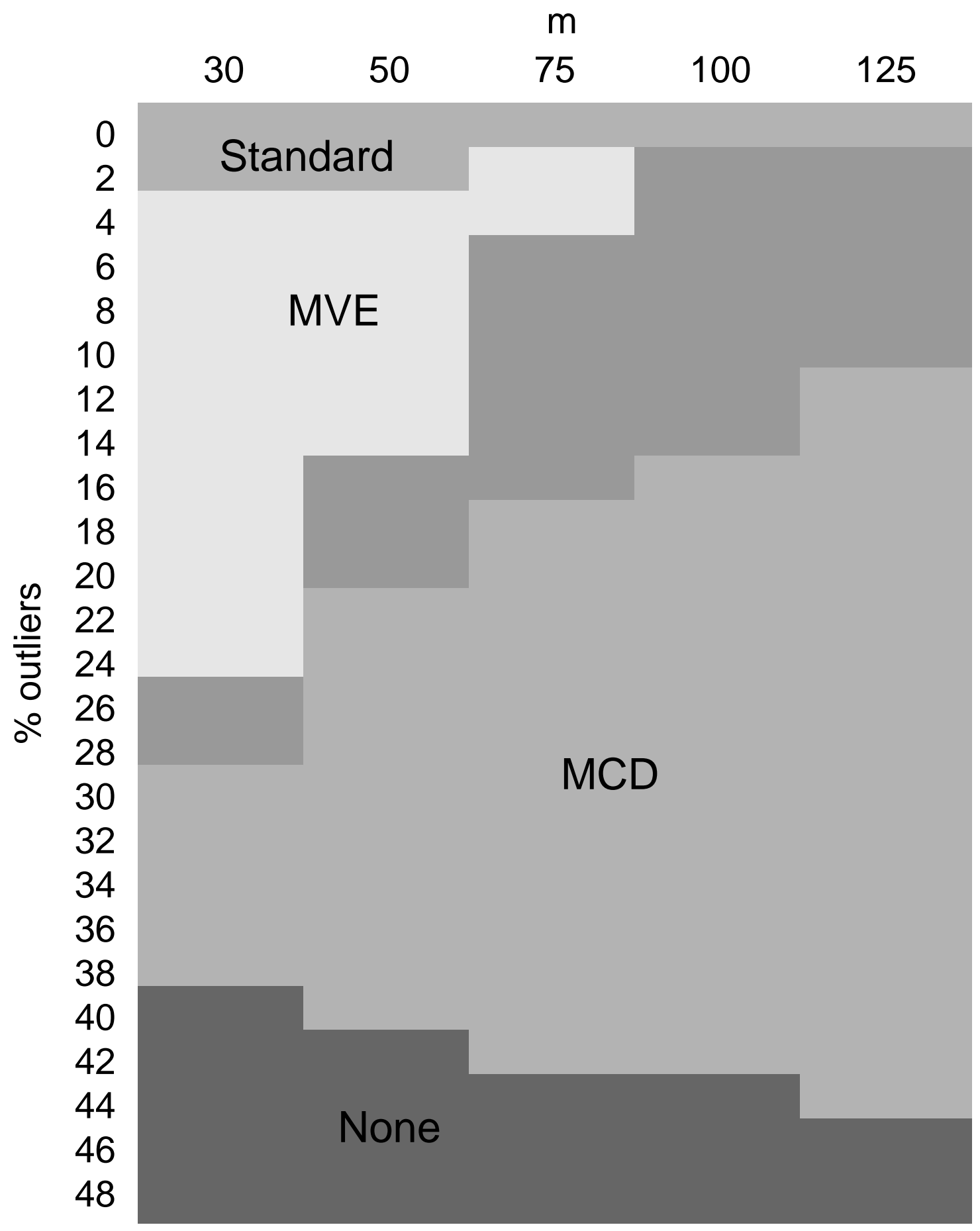

Figure 2: Summary of which estimator is preferred for where $p=2$. The unlabelled area is where the MVE and MCD perform equally well. 


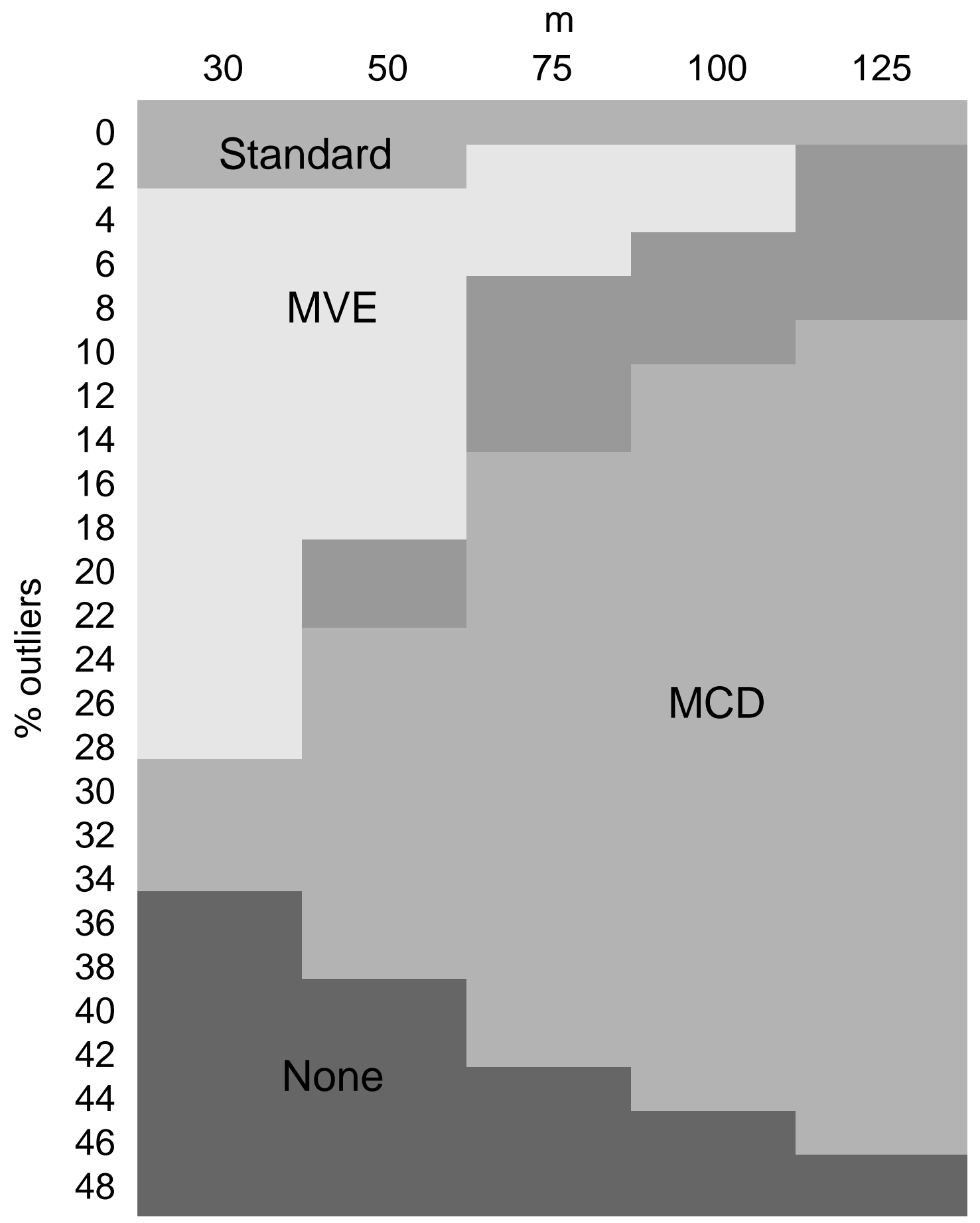

Figure 3: Summary of which estimator is preferred for where $p=3$. The unlabelled area is where the MVE and MCD perform equally well. 


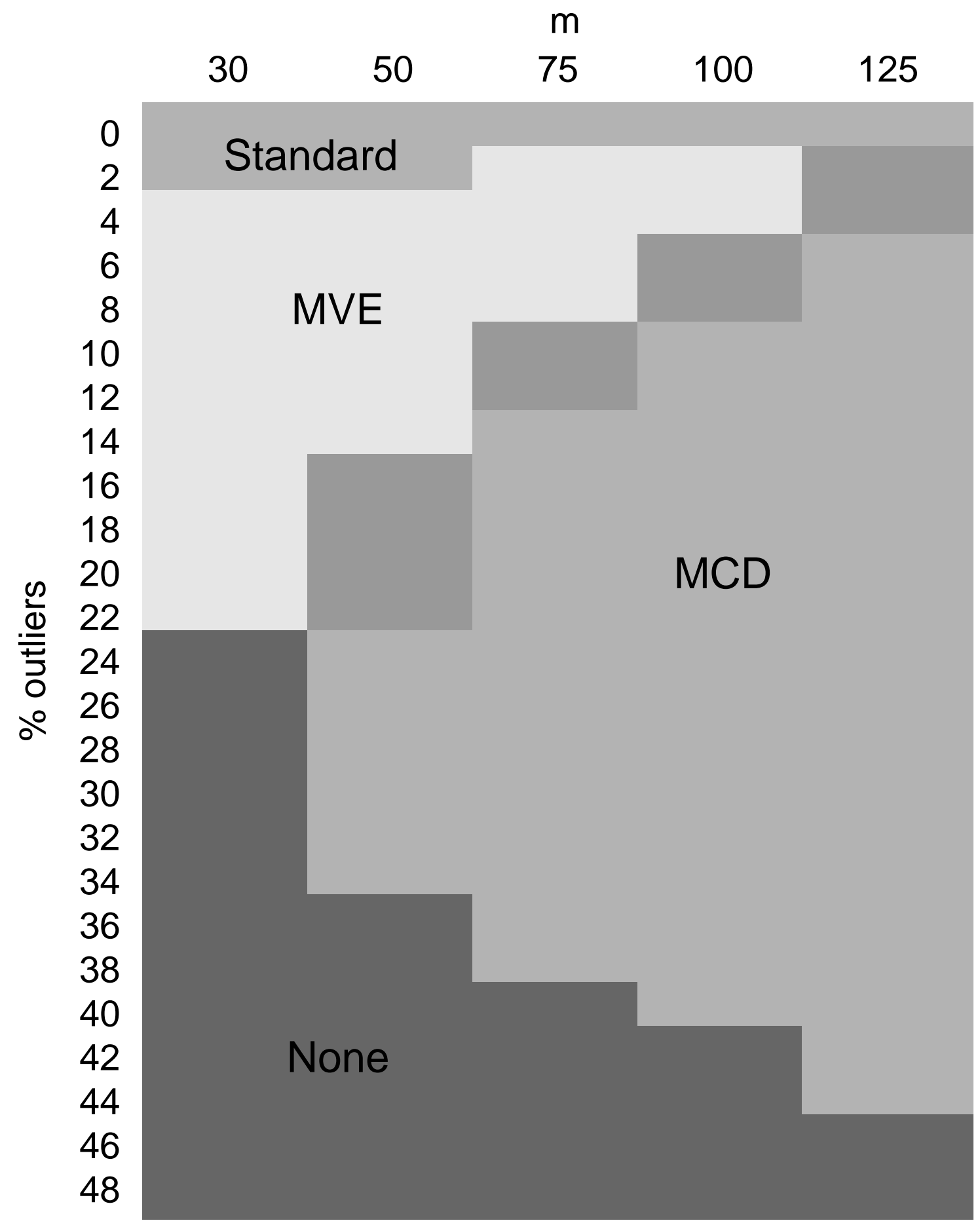

Figure 4: Summary of which estimator is preferred for where $p=5$. The unlabelled area is where the MVE and MCD perform equally well. 


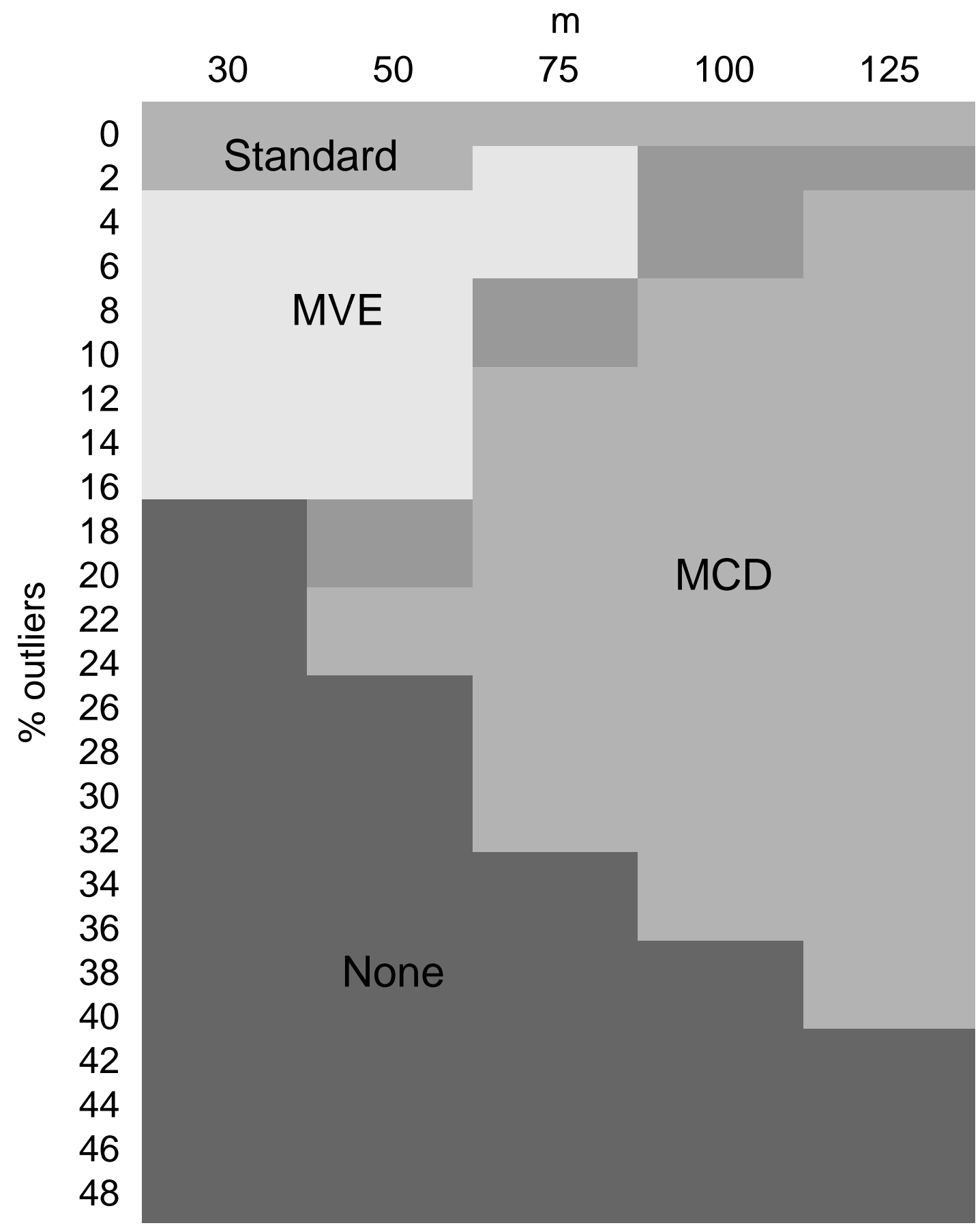

Figure 5: Summary of which estimator is preferred for where $p=7$. The unlabelled area is where the MVE and MCD perform equally well. 


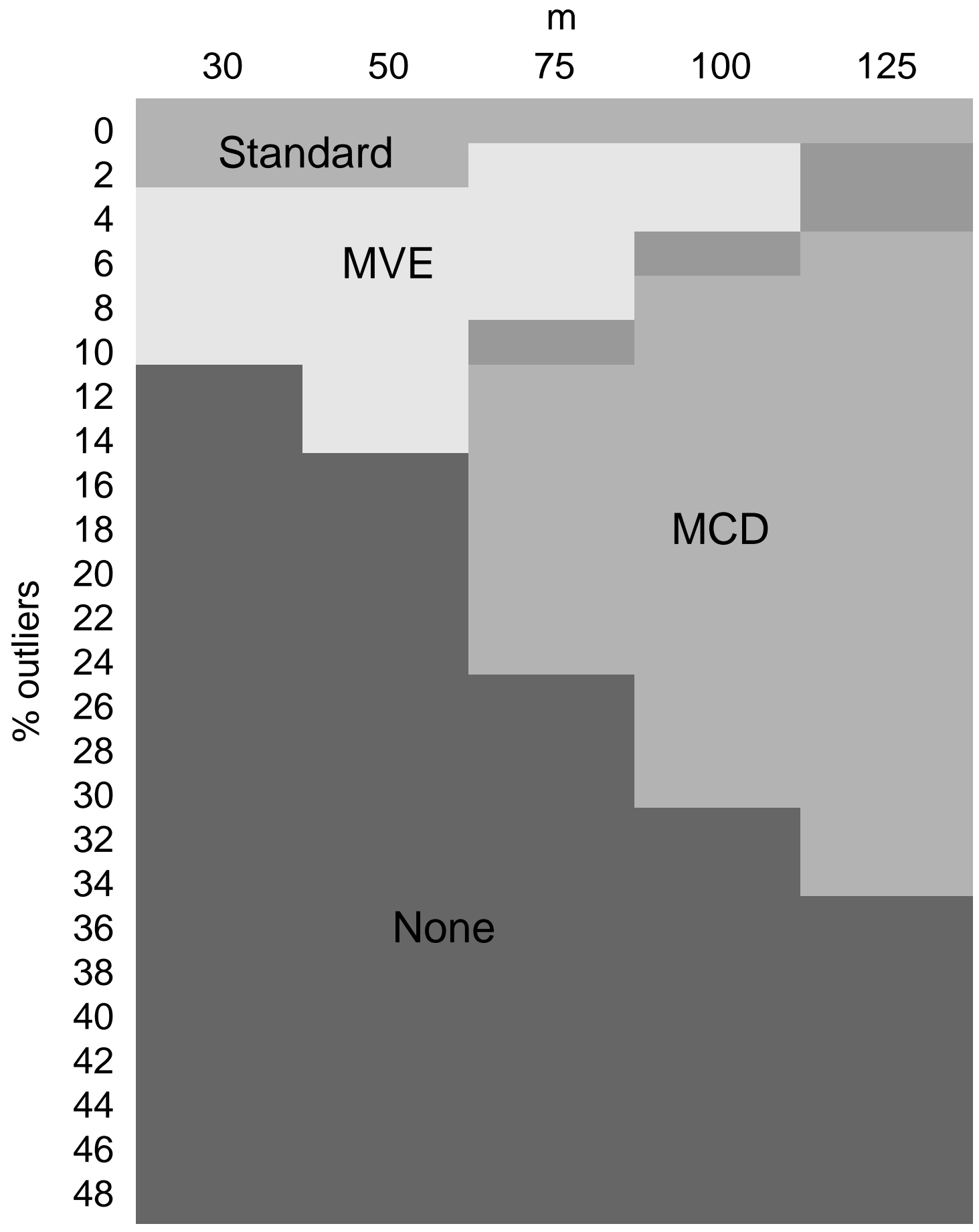

Figure 6: Summary of which estimator is preferred for where $p=10$. The unlabelled area is where the MVE and MCD perform equally well. 
estimate robustly (via S estimation or some other approach) and not be concerned with the deletion of outliers. So rather than focusing on the estimator that gives the best Phase I performance as was done here, the focus would be to find the estimator that gives the best Phase II performance.

\section{Conclusion}

It is important for Phase I multivariate control charts to be based on a high breakdown estimator in order to ensure that outliers are detected and that, as a result, the Phase II control limits will be meaningful. Both the MVE and MCD estimators are effective in detecting multiple outliers, but each is more advantageous for certain combinations of sample size and the number of outliers present. The MVE estimator is preferred for smaller sample sizes and a smaller percentage of outliers while the MCD is preferred for larger sample sizes and/or large percentages of outliers. The simulations and generated control limits presented here give useful guidelines about the situations for which high breakdown approach is most appropriate. 


\section{Appendix A - Tables of Control limits}

Table A . 1: Control limits for $T_{m v e, i}^{2}$ statistic obtained via subsampling to maintain an overall probability of signal $=0.05$ when the process is in control

\begin{tabular}{|c|c|c|c|c|c|c|c|c|c|}
\hline & \multicolumn{9}{|c|}{$\mathrm{p}$} \\
\hline $\mathrm{m}$ & 2 & 3 & 4 & 5 & 6 & 7 & 8 & 9 & 10 \\
\hline 20 & 30.15 & 38.48 & 63.28 & 62.83 & 97.05 & 89.44 & 149.47 & 140.90 & 284.29 \\
\hline 21 & 25.46 & 43.74 & 48.11 & 72.39 & 68.58 & 101.70 & 100.27 & 154.19 & 156.86 \\
\hline 22 & 28.70 & 35.21 & 54.51 & 54.34 & 75.19 & 75.16 & 107.58 & 107.21 & 167.24 \\
\hline 23 & 24.22 & 39.88 & 43.49 & 60.38 & 59.66 & 80.36 & 82.58 & 116.23 & 118.72 \\
\hline 24 & 26.50 & 33.40 & 47.82 & 49.27 & 64.11 & 65.27 & 87.01 & 89.92 & 126.12 \\
\hline 25 & 23.61 & 36.44 & 39.98 & 53.04 & 53.40 & 69.42 & 71.86 & 95.80 & 100.59 \\
\hline 26 & 25.72 & 31.76 & 43.08 & 45.71 & 57.23 & 58.87 & 74.65 & 79.58 & 105.12 \\
\hline 27 & 23.18 & 34.34 & 37.64 & 47.94 & 49.41 & 62.19 & 65.45 & 83.00 & 88.05 \\
\hline 28 & 24.88 & 30.48 & 40.20 & 42.68 & 52.25 & 54.66 & 67.87 & 72.59 & 91.04 \\
\hline 29 & 22.91 & 32.66 & 36.01 & 44.97 & 46.83 & 56.67 & 60.81 & 74.57 & 79.35 \\
\hline 30 & 24.31 & 29.52 & 37.97 & 40.21 & 48.48 & 51.65 & 62.15 & 67.41 & 81.74 \\
\hline 31 & 22.50 & 31.45 & 34.42 & 42.00 & 44.42 & 53.23 & 57.06 & 68.67 & 73.84 \\
\hline 32 & 23.70 & 28.74 & 36.37 & 38.84 & 46.18 & 49.00 & 58.47 & 63.16 & 75.60 \\
\hline 33 & 22.26 & 30.32 & 33.39 & 40.13 & 42.75 & 50.21 & 53.97 & 64.14 & 69.49 \\
\hline 34 & 23.48 & 28.02 & 34.70 & 37.41 & 44.13 & 46.78 & 55.51 & 60.12 & 70.52 \\
\hline 35 & 22.03 & 29.35 & 32.46 & 38.60 & 41.24 & 48.07 & 52.14 & 60.81 & 65.62 \\
\hline 36 & 23.05 & 27.58 & 33.54 & 36.27 & 42.18 & 45.46 & 52.86 & 57.84 & 67.13 \\
\hline 37 & 21.79 & 28.71 & 31.67 & 37.43 & 40.11 & 46.58 & 50.49 & 58.23 & 63.12 \\
\hline 38 & 22.71 & 26.98 & 32.69 & 35.43 & 41.01 & 44.50 & 51.26 & 55.38 & 63.86 \\
\hline 39 & 21.58 & 28.04 & 30.95 & 36.24 & 39.22 & 45.29 & 48.84 & 56.33 & 60.83 \\
\hline 40 & 22.48 & 26.50 & 32.09 & 34.84 & 40.04 & 43.02 & 49.61 & 53.67 & 61.23 \\
\hline 41 & 21.52 & 27.51 & 30.68 & 35.43 & 38.44 & 43.81 & 47.65 & 54.35 & 58.71 \\
\hline 42 & 22.48 & 26.19 & 31.28 & 34.00 & 39.26 & 42.23 & 48.24 & 52.15 & 59.35 \\
\hline 43 & 21.27 & 26.98 & 30.10 & 34.88 & 37.65 & 42.88 & 46.58 & 52.76 & 57.20 \\
\hline 44 & 22.02 & 25.90 & 30.80 & 33.76 & 38.33 & 41.59 & 47.10 & 50.84 & 57.59 \\
\hline 45 & 21.25 & 26.61 & 29.68 & 34.16 & 36.94 & 42.10 & 45.85 & 51.60 & 55.84 \\
\hline 46 & 21.92 & 25.57 & 30.34 & 32.99 & 37.72 & 40.85 & 46.27 & 50.00 & 56.18 \\
\hline 47 & 21.20 & 26.32 & 29.29 & 33.67 & 36.54 & 41.39 & 44.85 & 50.70 & 54.70 \\
\hline 48 & 21.78 & 25.38 & 29.87 & 32.64 & 37.02 & 40.22 & 45.35 & 49.32 & 54.89 \\
\hline 49 & 21.08 & 25.90 & 28.91 & 33.21 & 36.05 & 40.58 & 44.23 & 49.47 & 53.49 \\
\hline 50 & 21.68 & 25.24 & 29.46 & 32.25 & 36.49 & 39.58 & 44.56 & 48.20 & 53.75 \\
\hline
\end{tabular}


Table A . 2: Control limits for $T_{m v e, i}^{2}$ statistic obtained via subsampling to maintain an overall probability of signal $=0.05$ when the process is in control

\begin{tabular}{|c|c|c|c|c|c|c|c|c|c|}
\hline & \multicolumn{4}{|c|}{$\mathrm{p}$} & \multicolumn{5}{|c|}{$\mathrm{p}$} \\
\hline $\mathrm{m}$ & 2 & 3 & 4 & 5 & $\mathrm{~m}$ & 2 & 3 & 4 & 5 \\
\hline 51 & 20.97 & 25.86 & 28.64 & 32.73 & 76 & 20.70 & 23.76 & 27.09 & 29.80 \\
\hline 52 & 21.53 & 24.96 & 29.12 & 31.99 & 77 & 20.31 & 24.00 & 26.70 & 30.01 \\
\hline 53 & 20.90 & 25.40 & 28.39 & 32.33 & 78 & 20.66 & 23.68 & 26.91 & 29.70 \\
\hline 54 & 21.33 & 24.86 & 28.90 & 31.64 & 79 & 20.32 & 23.99 & 26.66 & 30.00 \\
\hline 55 & 20.77 & 25.26 & 28.22 & 32.05 & 80 & 20.69 & 23.62 & 26.89 & 29.68 \\
\hline 56 & 21.27 & 24.58 & 28.54 & 31.44 & 81 & 20.37 & 23.85 & 26.65 & 29.95 \\
\hline 57 & 20.72 & 25.06 & 27.95 & 31.73 & 82 & 20.69 & 23.62 & 26.82 & 29.51 \\
\hline 58 & 21.24 & 24.52 & 28.35 & 31.14 & 83 & 20.34 & 23.76 & 26.52 & 29.79 \\
\hline 59 & 20.62 & 25.00 & 27.79 & 31.59 & 84 & 20.57 & 23.49 & 26.73 & 29.52 \\
\hline 60 & 21.16 & 24.34 & 28.16 & 30.97 & 85 & 20.29 & 23.75 & 26.54 & 29.67 \\
\hline 61 & 20.64 & 24.80 & 27.67 & 31.26 & 86 & 20.56 & 23.46 & 26.70 & 29.45 \\
\hline 62 & 21.08 & 24.29 & 28.01 & 30.78 & 87 & 20.25 & 23.72 & 26.41 & 29.62 \\
\hline 63 & 20.60 & 24.66 & 27.40 & 31.06 & 88 & 20.58 & 23.45 & 26.67 & 29.40 \\
\hline 64 & 21.09 & 24.21 & 27.84 & 30.60 & 89 & 20.30 & 23.65 & 26.38 & 29.51 \\
\hline 65 & 20.59 & 24.61 & 27.29 & 30.88 & 90 & 20.57 & 23.41 & 26.63 & 29.21 \\
\hline 66 & 20.94 & 24.06 & 27.60 & 30.42 & 91 & 20.33 & 23.63 & 26.35 & 29.50 \\
\hline 67 & 20.45 & 24.46 & 27.24 & 30.69 & 92 & 20.50 & 23.48 & 26.49 & 29.26 \\
\hline 68 & 20.91 & 23.96 & 27.46 & 30.29 & 93 & 20.24 & 23.64 & 26.31 & 29.40 \\
\hline 69 & 20.47 & 24.38 & 27.10 & 30.55 & 94 & 20.48 & 23.28 & 26.51 & 29.18 \\
\hline 70 & 20.84 & 23.95 & 27.43 & 30.12 & 95 & 20.23 & 23.51 & 26.23 & 29.31 \\
\hline 71 & 20.44 & 24.30 & 27.03 & 30.45 & 96 & 20.46 & 23.27 & 26.41 & 29.12 \\
\hline 72 & 20.79 & 23.85 & 27.28 & 30.11 & 97 & 20.23 & 23.56 & 26.22 & 29.25 \\
\hline 73 & 20.42 & 24.16 & 26.90 & 30.28 & 98 & 20.48 & 23.32 & 26.38 & 29.12 \\
\hline 74 & 20.71 & 23.76 & 27.14 & 29.98 & 99 & 20.23 & 23.51 & 26.21 & 29.23 \\
\hline 75 & 20.40 & 24.01 & 26.82 & 30.15 & 100 & 20.40 & 23.25 & 26.35 & 29.08 \\
\hline
\end{tabular}


Table A . 3: Control limits for $T_{m c d, i}^{2}$ statistic obtained via the FAST-MCD algorithm to maintain an overall probability of signal $=0.05$ when the process is in control

\begin{tabular}{|c|c|c|c|c|c|c|c|c|c|}
\hline \multirow[b]{2}{*}{$\mathrm{m}$} & \multicolumn{9}{|c|}{$\mathrm{p}$} \\
\hline & 2 & 3 & 4 & 5 & 6 & 7 & 8 & 9 & 10 \\
\hline 20 & 116.35 & 221.31 & 512.57 & 573.00 & 1263.75 & 1261.29 & 3216.16 & 2732.59 & 8607.95 \\
\hline 21 & 101.69 & 267.12 & 356.97 & 732.92 & 780.43 & 1749.17 & 1635.04 & 4263.75 & 3475.45 \\
\hline 22 & 96.48 & 190.82 & 426.29 & 494.98 & 1001.39 & 1021.63 & 2306.46 & 2099.58 & 5394.73 \\
\hline 23 & 88.45 & 225.37 & 312.60 & 614.28 & 658.21 & 1338.25 & 1316.79 & 2925.82 & 2617.15 \\
\hline 24 & 82.50 & 165.93 & 363.30 & 433.36 & 824.27 & 854.21 & 1717.70 & 1654.24 & 3699.36 \\
\hline 25 & 76.88 & 191.09 & 276.33 & 524.46 & 573.24 & 1080.57 & 1081.13 & 2153.21 & 2006.16 \\
\hline 26 & 71.17 & 142.78 & 310.01 & 386.96 & 689.36 & 729.03 & 1358.16 & 1343.84 & 2640.49 \\
\hline 27 & 67.76 & 161.40 & 244.43 & 451.12 & 507.49 & 887.25 & 919.94 & 1676.68 & 1606.62 \\
\hline 28 & 63.58 & 124.24 & 266.56 & 341.02 & 592.22 & 638.91 & 1103.96 & 1118.05 & 2010.77 \\
\hline 29 & 60.79 & 140.21 & 218.91 & 397.85 & 453.10 & 752.13 & 784.03 & 1328.16 & 1330.95 \\
\hline 30 & 57.87 & 109.75 & 229.62 & 306.61 & 521.58 & 565.05 & 920.97 & 938.37 & 1584.42 \\
\hline 31 & 56.06 & 121.26 & 192.12 & 348.47 & 403.29 & 649.23 & 682.26 & 1100.83 & 1109.26 \\
\hline 32 & 52.81 & 96.93 & 198.93 & 273.13 & 461.13 & 502.58 & 791.71 & 811.53 & 1278.29 \\
\hline 33 & 51.65 & 106.74 & 169.80 & 306.25 & 368.58 & 567.87 & 603.86 & 927.22 & 943.58 \\
\hline 34 & 48.73 & 87.04 & 174.05 & 243.99 & 402.76 & 446.01 & 678.92 & 709.17 & 1064.44 \\
\hline 35 & 47.82 & 94.92 & 151.74 & 272.18 & 331.76 & 502.06 & 535.50 & 787.65 & 798.08 \\
\hline 36 & 45.88 & 78.60 & 151.54 & 219.12 & 359.95 & 403.76 & 592.05 & 618.53 & 893.32 \\
\hline 37 & 45.39 & 85.04 & 135.32 & 240.93 & 299.32 & 446.20 & 478.61 & 685.69 & 703.16 \\
\hline 38 & 43.28 & 71.83 & 134.45 & 195.70 & 320.15 & 368.05 & 523.59 & 550.52 & 768.13 \\
\hline 39 & 42.95 & 77.90 & 121.48 & 214.69 & 273.21 & 398.36 & 435.75 & 598.72 & 621.28 \\
\hline 40 & 40.83 & 66.67 & 118.92 & 176.28 & 283.51 & 331.61 & 466.44 & 493.09 & 670.10 \\
\hline 41 & 40.89 & 71.31 & 110.40 & 190.07 & 245.31 & 358.95 & 391.38 & 532.95 & 552.36 \\
\hline 42 & 39.16 & 62.05 & 108.37 & 158.55 & 255.07 & 300.85 & 417.22 & 444.66 & 591.07 \\
\hline 43 & 39.04 & 66.12 & 100.11 & 171.10 & 223.88 & 325.15 & 355.00 & 476.19 & 497.57 \\
\hline 44 & 37.40 & 58.53 & 98.07 & 142.79 & 229.56 & 273.68 & 377.42 & 401.43 & 527.47 \\
\hline 45 & 37.57 & 61.83 & 91.20 & 153.44 & 203.37 & 294.03 & 325.84 & 432.77 & 446.93 \\
\hline 46 & 35.95 & 55.21 & 90.13 & 129.36 & 205.50 & 251.08 & 340.95 & 367.12 & 474.81 \\
\hline 47 & 35.91 & 58.29 & 85.00 & 137.97 & 184.30 & 266.10 & 297.59 & 388.44 & 408.06 \\
\hline 48 & 34.83 & 52.52 & 82.86 & 118.33 & 185.14 & 228.42 & 311.98 & 337.64 & 427.32 \\
\hline 49 & 34.96 & 55.05 & 78.76 & 125.40 & 170.19 & 240.78 & 272.27 & 351.29 & 373.57 \\
\hline 50 & 33.63 & 50.05 & 76.83 & 109.48 & 166.78 & 208.99 & 281.82 & 307.01 & 388.70 \\
\hline 51 & 34.00 & 52.21 & 74.30 & 114.33 & 153.54 & 221.02 & 251.74 & 322.26 & 340.80 \\
\hline 52 & 32.80 & 47.63 & 72.24 & 100.22 & 152.20 & 190.70 & 260.23 & 284.55 & 353.93 \\
\hline 53 & 33.00 & 49.94 & 69.68 & 104.77 & 141.09 & 199.71 & 234.03 & 297.29 & 316.36 \\
\hline 54 & 32.08 & 45.89 & 68.06 & 93.16 & 138.70 & 175.65 & 236.04 & 261.73 & 326.26 \\
\hline 55 & 32.51 & 47.86 & 65.91 & 97.77 & 129.80 & 185.02 & 215.83 & 273.59 & 291.71 \\
\hline 56 & 31.44 & 44.24 & 64.20 & 86.75 & 128.02 & 160.04 & 216.92 & 242.96 & 299.15 \\
\hline 57 & 31.42 & 46.12 & 62.25 & 90.67 & 119.58 & 169.09 & 197.30 & 250.11 & 270.43 \\
\hline 58 & 30.60 & 43.00 & 60.88 & 81.39 & 117.37 & 149.04 & 198.86 & 224.10 & 277.98 \\
\hline 59 & 30.85 & 44.21 & 59.22 & 84.91 & 111.41 & 154.91 & 182.85 & 233.49 & 254.14 \\
\hline 60 & 29.93 & 41.51 & 58.09 & 76.89 & 108.83 & 137.20 & 182.80 & 208.72 & 257.63 \\
\hline
\end{tabular}


Table A . 4: Control limits for the $T_{m c d, i}^{2}$ statistic obtained via the FAST-MCD algorithm to maintain an overall probability of signal $=0.05$ when the process is in control

\begin{tabular}{|c|c|c|c|c|c|c|c|c|c|}
\hline & \multicolumn{9}{|c|}{$\mathrm{p}$} \\
\hline $\mathrm{m}$ & 2 & 3 & 4 & 5 & 6 & 7 & 8 & 9 & 10 \\
\hline 61 & 30.37 & 42.94 & 56.89 & 79.90 & 103.46 & 142.42 & 170.75 & 217.32 & 236.53 \\
\hline 62 & 29.60 & 40.05 & 55.66 & 72.70 & 100.76 & 128.10 & 169.24 & 193.12 & 240.25 \\
\hline 63 & 29.81 & 41.60 & 54.41 & 75.19 & 96.07 & 132.49 & 158.53 & 200.15 & 219.66 \\
\hline 64 & 28.97 & 39.30 & 53.53 & 69.09 & 94.22 & 118.68 & 157.60 & 180.96 & 222.52 \\
\hline 65 & 29.31 & 40.50 & 52.23 & 71.24 & 90.69 & 123.39 & 147.74 & 186.37 & 206.70 \\
\hline 66 & 28.52 & 38.19 & 51.52 & 65.55 & 88.53 & 110.76 & 146.46 & 169.88 & 209.14 \\
\hline 67 & 28.78 & 39.42 & 50.62 & 67.75 & 85.59 & 114.25 & 137.55 & 174.27 & 193.82 \\
\hline 68 & 28.20 & 37.55 & 49.63 & 62.58 & 83.68 & 103.68 & 136.62 & 158.56 & 193.39 \\
\hline 69 & 28.43 & 38.56 & 49.04 & 64.45 & 81.00 & 108.00 & 128.53 & 162.14 & 181.61 \\
\hline 70 & 27.83 & 36.53 & 48.24 & 60.42 & 79.45 & 97.71 & 126.77 & 148.48 & 181.76 \\
\hline 71 & 28.03 & 37.58 & 47.41 & 61.90 & 77.35 & 100.50 & 120.42 & 151.79 & 170.44 \\
\hline 72 & 27.50 & 35.87 & 46.58 & 58.14 & 75.28 & 92.39 & 118.68 & 139.10 & 170.02 \\
\hline 73 & 27.69 & 36.77 & 46.03 & 59.70 & 73.34 & 94.81 & 113.96 & 142.75 & 161.94 \\
\hline 74 & 27.05 & 35.22 & 45.36 & 56.10 & 72.34 & 87.58 & 112.12 & 131.52 & 160.34 \\
\hline 75 & 27.38 & 35.89 & 45.00 & 57.47 & 70.37 & 89.91 & 107.88 & 133.60 & 152.33 \\
\hline 76 & 26.85 & 34.45 & 44.20 & 53.92 & 68.94 & 83.38 & 105.06 & 123.58 & 151.70 \\
\hline 77 & 27.10 & 35.30 & 43.74 & 55.49 & 67.63 & 84.98 & 101.60 & 125.70 & 144.50 \\
\hline 78 & 26.57 & 34.19 & 43.17 & 52.45 & 66.11 & 79.10 & 99.23 & 116.99 & 142.80 \\
\hline 79 & 26.76 & 34.80 & 42.70 & 53.80 & 64.74 & 80.83 & 95.81 & 118.40 & 136.02 \\
\hline 80 & 26.24 & 33.46 & 42.03 & 50.93 & 63.46 & 75.95 & 94.29 & 110.74 & 134.38 \\
\hline 81 & 26.67 & 34.10 & 41.72 & 52.00 & 62.33 & 77.57 & 92.27 & 112.47 & 128.79 \\
\hline 82 & 26.02 & 32.99 & 41.20 & 49.55 & 61.36 & 72.67 & 90.02 & 105.60 & 127.13 \\
\hline 83 & 26.25 & 33.71 & 41.16 & 50.67 & 60.19 & 74.76 & 87.58 & 107.04 & 123.17 \\
\hline 84 & 25.84 & 32.56 & 40.45 & 48.36 & 59.59 & 70.01 & 85.75 & 99.78 & 120.54 \\
\hline 85 & 26.05 & 33.11 & 40.34 & 49.19 & 58.18 & 71.34 & 83.71 & 102.45 & 116.80 \\
\hline 86 & 25.61 & 32.07 & 39.66 & 47.17 & 57.70 & 67.48 & 82.18 & 94.92 & 114.90 \\
\hline 87 & 25.90 & 32.71 & 39.58 & 47.94 & 56.54 & 68.76 & 80.03 & 97.42 & 111.43 \\
\hline 88 & 25.50 & 31.90 & 38.79 & 46.19 & 55.73 & 65.49 & 78.83 & 91.25 & 109.30 \\
\hline 89 & 25.70 & 32.36 & 38.75 & 46.82 & 54.98 & 66.34 & 77.46 & 92.95 & 106.56 \\
\hline 90 & 25.24 & 31.45 & 38.26 & 45.19 & 54.11 & 63.28 & 75.59 & 87.85 & 104.28 \\
\hline 91 & 25.53 & 31.93 & 38.17 & 46.07 & 53.68 & 64.00 & 74.57 & 89.18 & 101.58 \\
\hline 92 & 25.12 & 31.07 & 37.77 & 44.15 & 52.94 & 61.22 & 73.18 & 84.42 & 99.39 \\
\hline 93 & 25.37 & 31.59 & 37.50 & 45.08 & 52.28 & 62.35 & 71.91 & 85.44 & 97.31 \\
\hline 94 & 24.93 & 30.73 & 37.17 & 43.48 & 51.37 & 59.57 & 70.76 & 81.17 & 95.86 \\
\hline 95 & 25.12 & 31.17 & 37.14 & 44.17 & 51.16 & 60.49 & 69.53 & 81.71 & 93.73 \\
\hline 96 & 24.76 & 30.47 & 36.56 & 42.67 & 50.50 & 58.00 & 68.21 & 78.23 & 92.06 \\
\hline 97 & 25.03 & 30.83 & 36.51 & 43.29 & 50.09 & 58.80 & 67.09 & 79.56 & 89.75 \\
\hline 98 & 24.60 & 30.15 & 36.18 & 42.05 & 49.29 & 56.77 & 66.18 & 75.89 & 88.54 \\
\hline 99 & 24.81 & 30.62 & 35.99 & 42.52 & 48.79 & 57.35 & 65.43 & 76.20 & 86.71 \\
\hline 100 & 24.47 & 29.90 & 35.68 & 41.30 & 48.28 & 55.25 & 64.40 & 73.49 & 85.16 \\
\hline
\end{tabular}




\section{Appendix B - Probability of Signal}
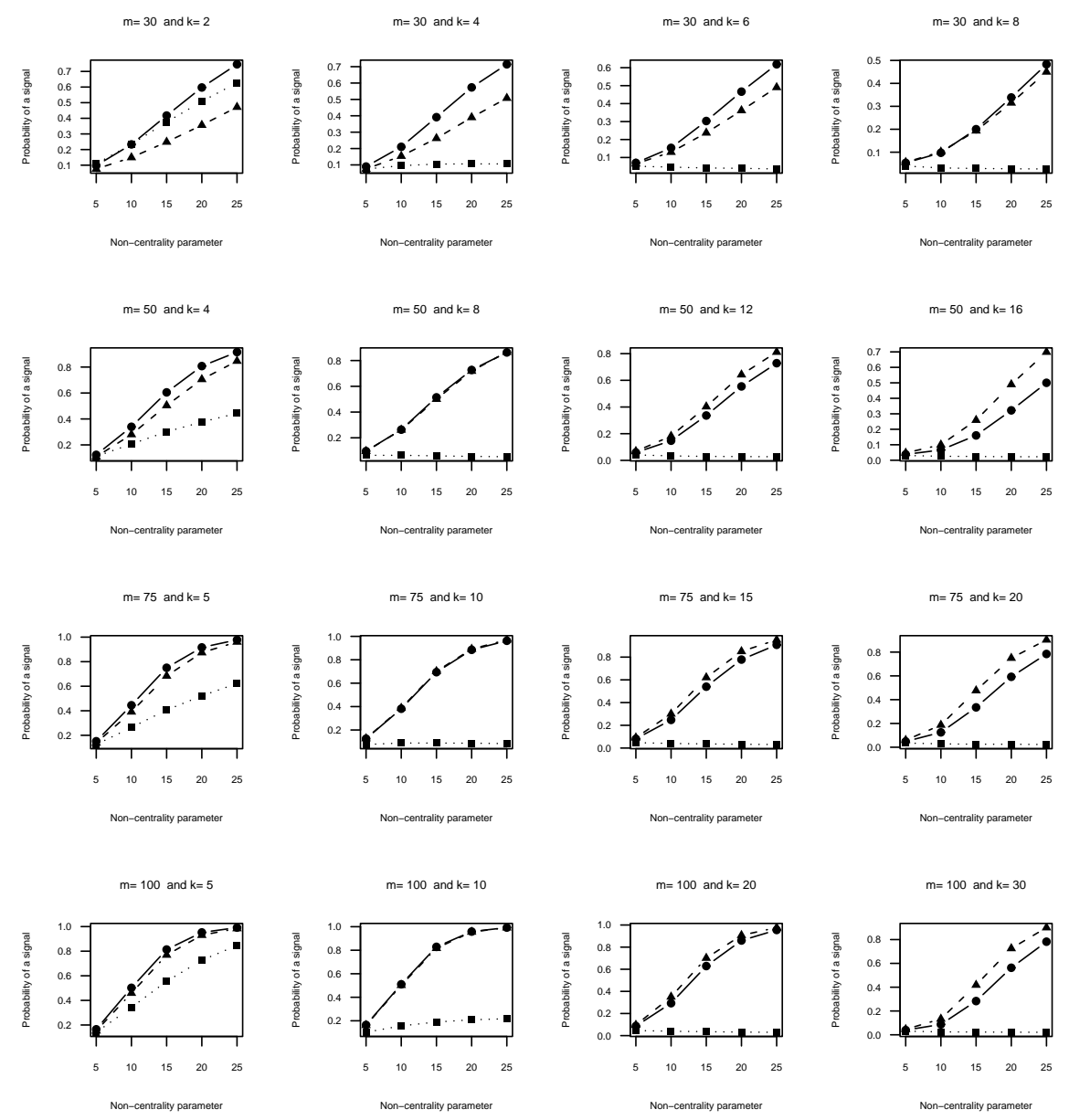

Figure B . 1: Probability of signal for various combinations of $m$ and $k$ for $p=2$. The circles and solid line correspond to the MVE, the triangles and dashed line correspond to the MCD, and the squares and dotted line correspond to the standard estimator. 
$\mathrm{m}=30$ and $\mathrm{k}=2$

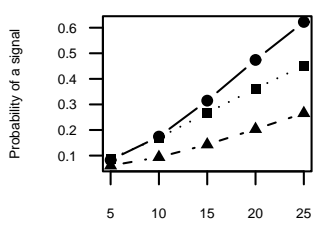

Non-centrality parameter

$\mathrm{m}=50$ and $\mathrm{k}=4$

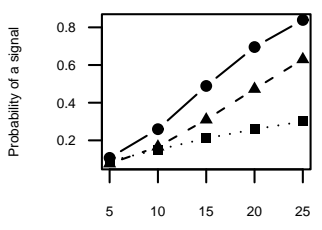

Non-centrality parameter

$m=75$ and $k=5$

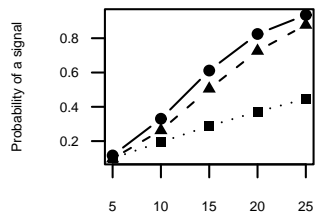

Non-centrality parameter

$\mathrm{m}=100$ and $\mathrm{k}=5$

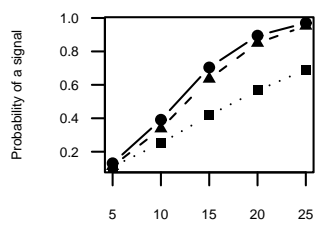

Non-centrality parameter $\mathrm{m}=30$ and $\mathrm{k}=4$

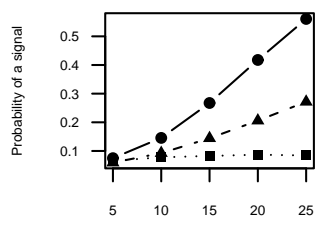

Non-centrality parameter

$\mathrm{m}=50$ and $\mathrm{k}=8$

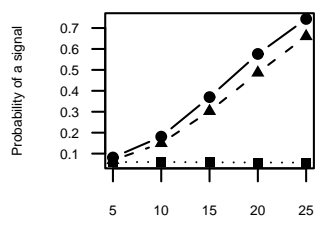

Non-centrality parameter

$\mathrm{m}=75$ and $\mathrm{k}=10$

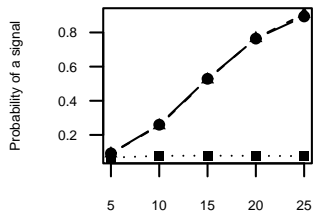

Non-centrality parameter

$m=100$ and $k=10$

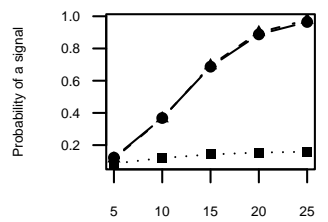

Non-centrality paramete $m=30$ and $k=6$

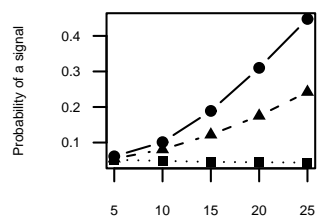

Non-centrality parameter

$\mathrm{m}=50$ and $\mathrm{k}=12$

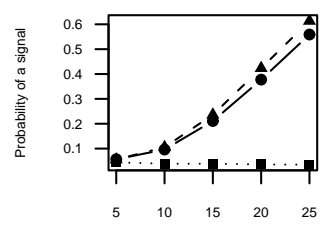

Non-centrality parameter

$\mathrm{m}=75$ and $\mathrm{k}=15$

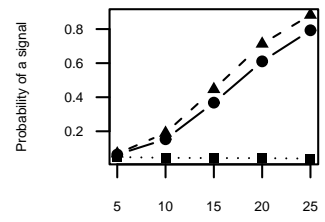

Non-centrality parameter

$m=100$ and $k=20$

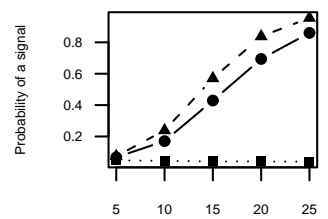

Non-centrality parameter $\mathrm{m}=30$ and $\mathrm{k}=8$

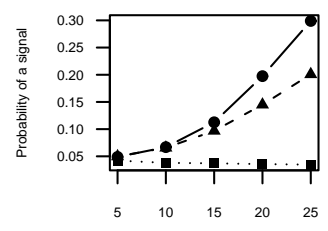

Non-centrality parameter

$\mathrm{m}=50$ and $\mathrm{k}=16$

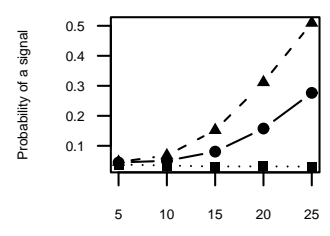

Non-centrality parameter

$m=75$ and $k=20$

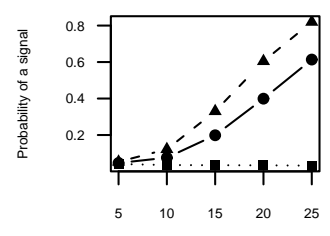

Non-centrality parameter

$m=100$ and $k=30$

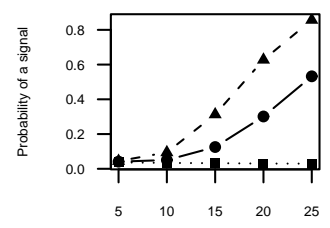

Non-centrality parameter

Figure B . 2: Probability of signal for various combinations of $m$ and $k$ for $p=3$. The circles and solid line correspond to the MVE, the triangles and dashed line correspond to the MCD, and the squares and dotted line correspond to the standard estimator. 
$\mathrm{m}=30$ and $\mathrm{k}=2$

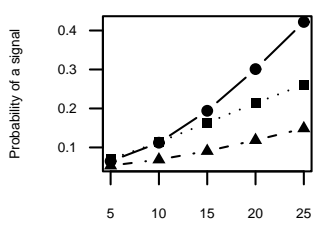

Non-centrality parameter

$\mathrm{m}=50$ and $\mathrm{k}=4$

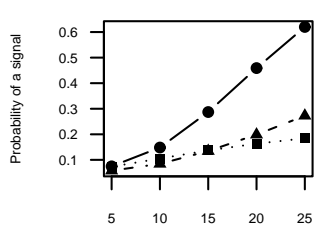

Non-centrality parameter

$m=75$ and $k=5$

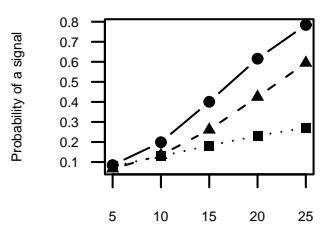

Non-centrality parameter

$\mathrm{m}=100$ and $\mathrm{k}=5$

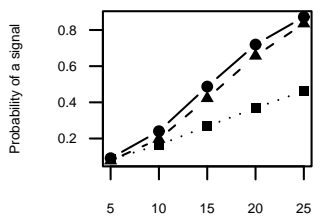

Non-centrality parameter $\mathrm{m}=30$ and $\mathrm{k}=4$

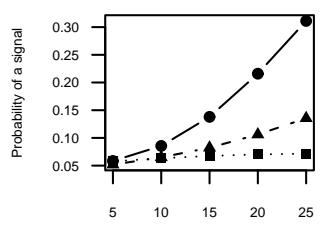

Non-centrality parameter

$m=50$ and $k=8$

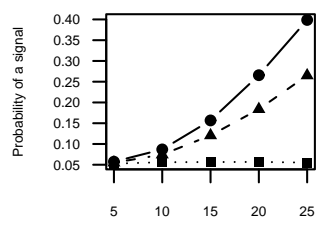

Non-centrality parameter

$\mathrm{m}=75$ and $\mathrm{k}=10$

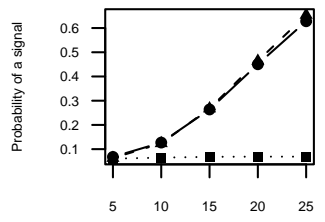

Non-centrality parameter

$m=100$ and $k=10$

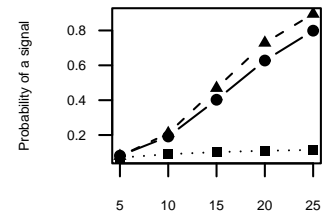

Non-centrality parameter $m=30$ and $k=6$

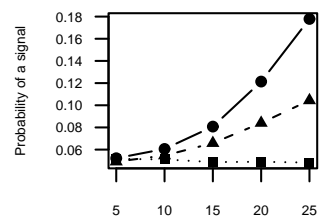

Non-centrality parameter

$\mathrm{m}=50$ and $\mathrm{k}=12$

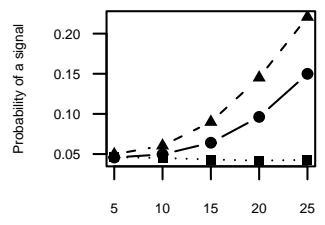

Non-centrality parameter

$\mathrm{m}=75$ and $\mathrm{k}=15$

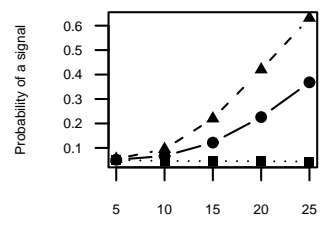

Non-centrality parameter

$\mathrm{m}=100$ and $\mathrm{k}=20$

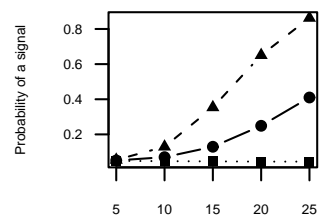

Non-centrality parameter $\mathrm{m}=30$ and $\mathrm{k}=8$

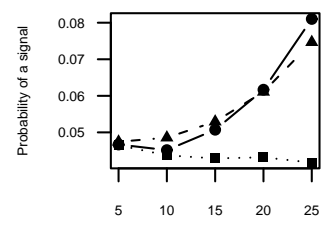

Non-centrality parameter

$m=50$ and $k=16$

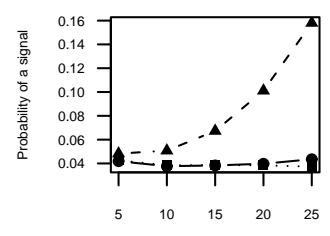

Non-centrality parameter

$m=75$ and $k=20$

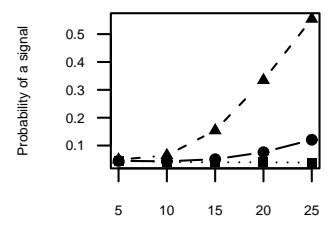

Non-centrality parameter

$m=100$ and $k=30$

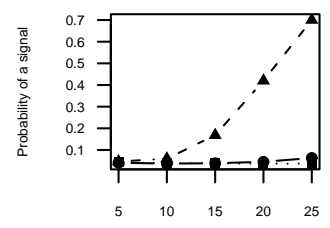

Non-centrality parameter

Figure B . 3: Probability of signal for various combinations of $m$ and $k$ for $p=5$. The circles and solid line correspond to the MVE, the triangles and dashed line correspond to the MCD, and the squares and dotted line correspond to the standard estimator. 
$\mathrm{m}=30$ and $\mathrm{k}=2$

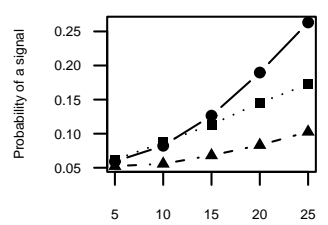

Non-centrality paramete

$\mathrm{m}=50$ and $\mathrm{k}=4$

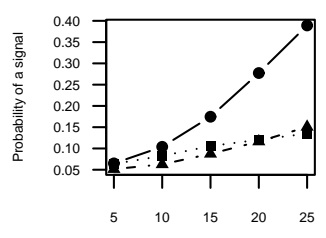

Non-centrality parameter

$m=75$ and $k=5$

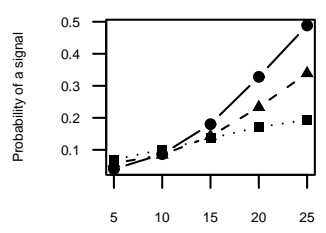

Non-centrality parameter

$m=100$ and $k=5$

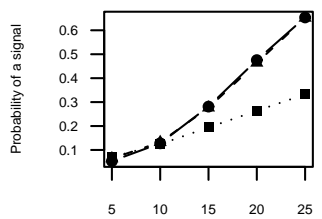

Non-centrality parameter $\mathrm{m}=30$ and $\mathrm{k}=4$

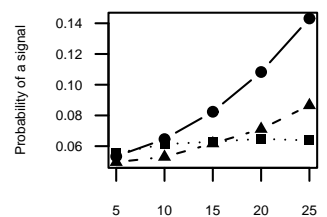

Non-centrality parameter

$\mathrm{m}=50$ and $\mathrm{k}=8$

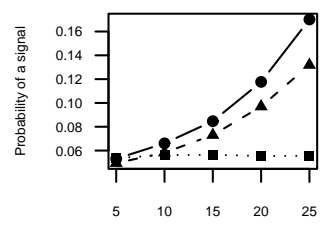

Non-centrality parameter

$m=75$ and $k=10$

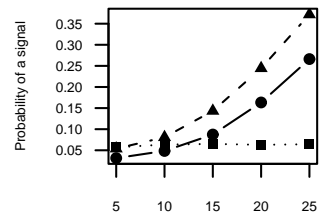

Non-centrality parameter

$m=100$ and $k=10$

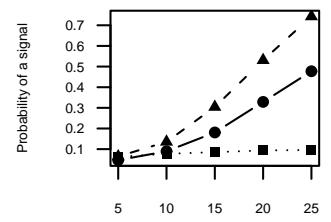

Non-centrality parameter $m=30$ and $k=6$

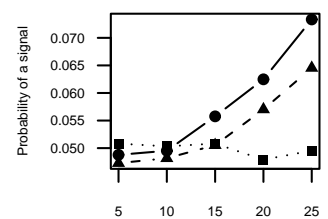

Non-centrality parameter

$\mathrm{m}=50$ and $\mathrm{k}=12$

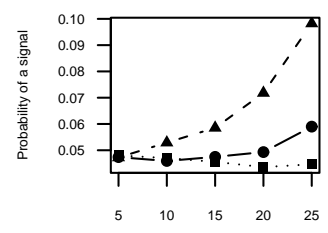

Non-centrality parameter

$m=75$ and $k=15$

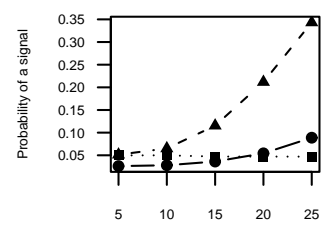

Non-centrality parameter

$\mathrm{m}=100$ and $\mathrm{k}=20$

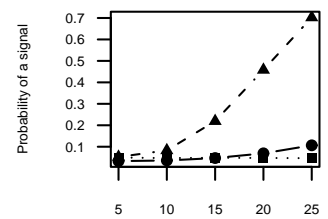

Non-centrality parameter $\mathrm{m}=30$ and $\mathrm{k}=8$

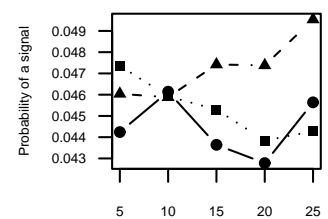

Non-centrality parameter

$\mathrm{m}=50$ and $\mathrm{k}=16$

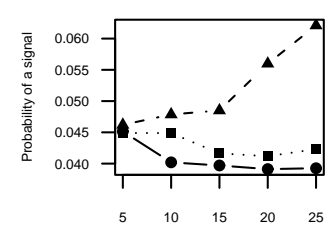

Non-centrality parameter

$\mathrm{m}=75$ and $\mathrm{k}=20$

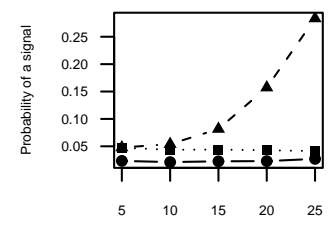

Non-centrality parameter

$m=100$ and $k=30$

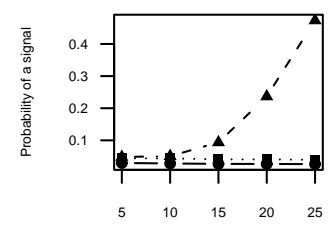

Non-centrality parameter

Figure B . 4: Probability of signal for various combinations of $m$ and $k$ for $p=7$. The circles and solid line correspond to the MVE, the triangles and dashed line correspond to the MCD, and the squares and dotted line correspond to the standard estimator. 
$\mathrm{m}=30$ and $\mathrm{k}=2$

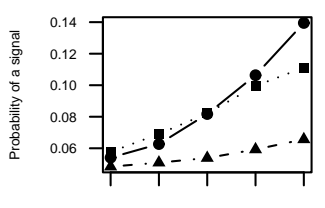

Non-centrality paramete

$m=50$ and $k=4$

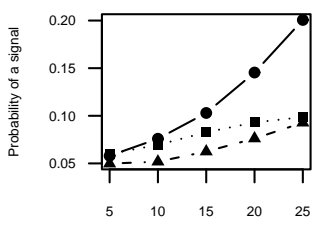

Non-centrality parameter

$m=75$ and $k=5$

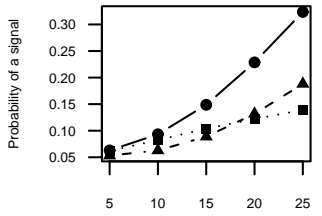

Non-centrality parameter

$m=100$ and $k=5$

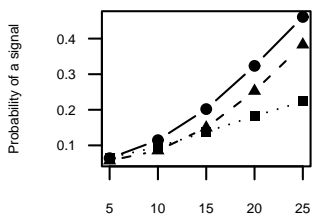

Non-centrality parameter $m=30$ and $k=4$

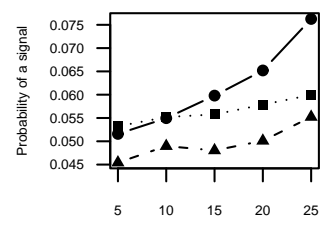

Non-centrality parameter

$m=50$ and $k=8$

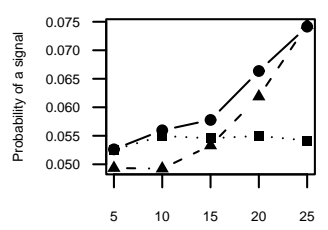

Non-centrality parameter

$\mathrm{m}=75$ and $\mathrm{k}=10$

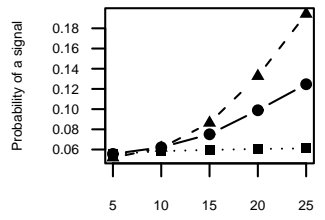

Non-centrality parameter

$m=100$ and $k=10$

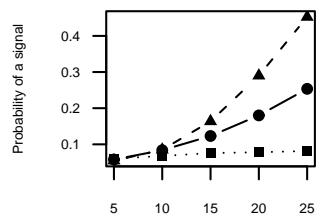

Non-centrality parameter $m=30$ and $k=6$

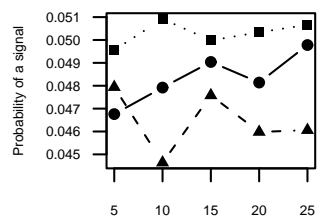

Non-centrality parameter

$\mathrm{m}=50$ and $\mathrm{k}=12$

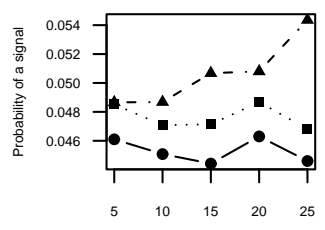

Non-centrality parameter

$\mathrm{m}=75$ and $\mathrm{k}=15$

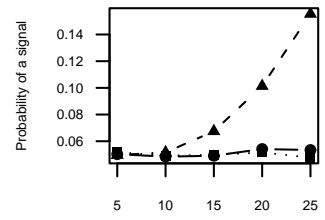

Non-centrality parameter

$\mathrm{m}=100$ and $\mathrm{k}=20$

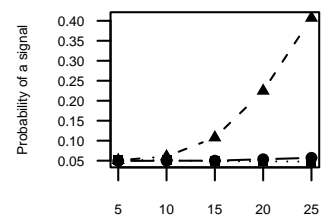

Non-centrality parameter $\mathrm{m}=30$ and $\mathrm{k}=8$

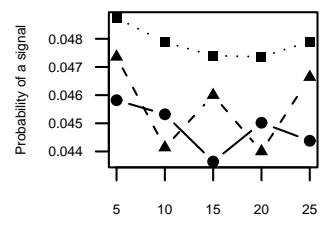

Non-centrality parameter

$\mathrm{m}=50$ and $\mathrm{k}=16$

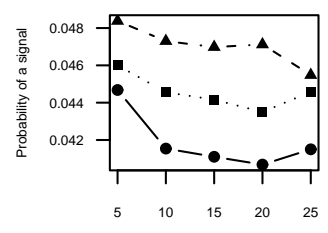

Non-centrality parameter

$\mathrm{m}=75$ and $\mathrm{k}=20$

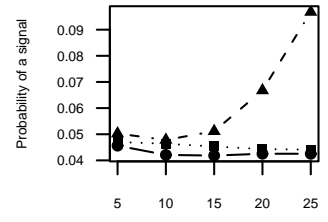

Non-centrality parameter

$m=100$ and $k=30$

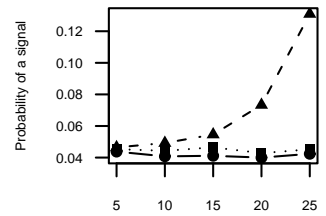

Non-centrality parameter

Figure B . 5: Probability of signal for various combinations of $m$ and $k$ for $p=10$. The circles and solid line correspond to the MVE, the triangles and dashed line correspond to the MCD, and the squares and dotted line correspond to the standard estimator. 


\section{REFERENCES}

Agullo, J. (1996). "Exact Iterative Computation of the Multivariate Minimum Volume Ellipsoid Estimator with a Branch and Bound Algorithm". in Proceedings in Computational Statistics, ed. A. Prat, Heidelberg:Physica-Verlag, pp. 175-180.

Agullo, J. (2001). "New Algorithms for Computing the Least Trimmed Squares Regression Estimator". Computational Statistics and Data Analysis 36, pp. 425-439.

Atkinson, A.C. (1993) "Stalactite Plots and Robust Estimation for the Detection of Multivariate Outliers" in Data Analysis and Robustness eds. S. Morgenthaler, E. Ronchetti, and W. Stahel, Basel:Birkhuser.

Atwood, C.L. (1973). "Sequences Converging to D-optimal Designs of Experiments". The Annals of Statistics 1, pp. 342-352.

Butler, R.W., Davies, P.L., and Jhun, M. (1993). "Asymptotics for the Minimum Covariance Determinant Estimator". The Annals of Statistics 21, pp. 1385-1400.

Cook, R.D. and Hawkins, D.M. (1990). Discussion of "Unmasking Multivariate Outlier and Leverage Points". Journal of the American Statistical Association 85, pp. 640644.

Cook, R.D., Hawkins, D.M., and Weisberg, S. (1993). "Exact Iterative Computation of the Robust Multivariate Minimum Volume Ellipsoid Estimator". Statistics and Probability Letters 16, pp. 213-218.

Croux, C. and Haesbroeck, G. (1997). "An Easy Way to Increase the Finite-Sample Efficiency of the Resampled Minimum Volume Ellipsoid Estimator". Computational Statistics \& Data Analysis 25, pp. 125-141.

Croux, C. and Haesbroeck, G. (2002). "A Note on Finite-Sample Efficiencies for the Minimum Volume Ellipsoid". Journal of Statistical Computation and Simulation 72 , pp. 585-596.

Davies, P.L. (1987). "Asymptotic Behavior of S-estimators of Multivariate Location Parameters and Dispersion Matrices". The Annals of Statistics 15, pp. 1269-1292.

Davies, P.L. (1992). "The Asymptotics of Rousseeuw's Minimum Volume Ellipsoid Estimator". The Annals of Statistics 20, pp. 1828-1843.

Davis, C.M. and Adams, B.M. (2005). "Robust Monitoring of Contaminated Data". Journal of Quality Technology 37, pp. 163-174.

Donoho, D.L. and Huber, P.J. (1983). The Notion of Breakdown Point. In A Festschrift for Erich Lehmann eds. Bickel, P., Doksum, K. and Hodges, J.L. Jr., pp. 157-184.

Hadi, A.S. (1992). "Identifying Multiple Outliers in Multivariate Data". The Journal of the Royal Statistical Society, Series B 54, pp. 761-777.

Hadi, A.S. (1994). "A Modification of a Method for the Detection of Outliers in Multivariate Samples". The Journal of the Royal Statistical Society, Series B 56, pp. 393-396. 
Hampel, F.R., Ronchetti, E.M., Rousseeuw, P.J., and Stahel, W.A. (1986) Robust Statistics: The Approach Based on Influence Functions John Wiley and Sons, New York, NY.

Hawkins, D.M. (1993). "A Feasible Solution Algorithm for the Minimum Volume Ellipsoid Estimator in Multivariate Data". Computational Statistics 8, pp. 95107.

Hawkins, D.M. (1994). "The Feasible Solution Algorithm for the Minimum Covariance Determinant Estimator in Multivariate Data". Computational Statistics \& Data Analysis 17, pp. 197-210.

Hawkins, D.M. and Olive, D.J. (1999). "Improved Feasible Solution Algorithms for High Breakdown Estimation". Computational Statistics 86 Data Analysis 30, pp. $1-11$.

Hawkins, D.M. and Olive, D.J. (2002). "Inconsistency of Resampling Algorithms for High-Breakdown Regression Estimators and a New Algorithm" (with discussion). Journal of the American Statistical Association 97, pp. 136-159.

Lopuhaä H.P. and Rousseeuw, P.J. (1991). "Breakdown Points of Affine Equivariant Estimators of Multivariate Location and Covariance Matrices". The Annals of Statistics 19, pp. 229-248.

Maronna, R.A. and Zamar, R.H. (2002). "Robust Estimates of Location and Dispersion for High-Dimensional Datasets". Technometrics 44, pp. 307-317.

Quesenberry, C.P. (2001). "The Multivariate Short-Run Snapshot Q Chart". Quality Engineering 13, pp. 679-683.

Rocke, D.M. (1989). "Robust Control Charts". Technometrics 31, pp. 173-184.

Rocke, D.M. (1992). " $\bar{X}_{Q}$ and $R_{Q}$ Charts: Robust Control Charts". Statistician 41, pp. $97-104$.

Rocke, D.M. and Woodruff, D.L. (1993). "Computation of Robust Estimates of Multivariate Location and Shape". Statistica Neerlandica 47, pp. 27-42.

Rocke, D.M. and Woodruff, D.L. (1996). "Identification of Outliers in Multivariate Data". Journal of the American Statistical Association 91, pp. 1047-1061.

Rocke, D.M. and Woodruff, D.L. (1997). "Robust Estimation of Multivariate Location and Shape". Journal of Statistical Planning and Inference 57, pp. 245-255.

Rousseeuw, P.J. (1984). "Least Median of Squares Regression". Journal of the American Statistical Association, 79, pp. 871-880.

Rousseeuw, P.J. and Leroy, A.M. (1987). Robust Regression and Outlier Detection. John Wiley \& Sons, New York, NY.

Rousseeuw, P.J. and Van Driessen, K. (1999). "A Fast Algorithm for the Minimum Covariance Determinant Estimator". Technometrics 41, pp. 212-223. 
Rousseeuw, P.J. and van Zomeren, B.C. (1990). "Unmasking Multivariate Outliers and Leverage Points". Journal of the American Statistical Association 85, pp. 633-639.

Rousseeuw, P.J. and van Zomeren, B.C. (1991). "Robust Distance: Simulations and Cutoff Values". in Directions in Robust Statistics and Diagnostics Part II eds. W. Stahel and S. Weisberg, New York: Springer-Verlag.

Sullivan, J.H. and Woodall, W.H. (1996). "A Comparison of Multivariate Control Charts for Individual Observations". Journal of Quality Technology 28, pp. 398408.

Tatum, L.G. (1997). "Robust Estimation of the Process Standard Deviation for Control Charts". Technometrics 39, pp. 127-141.

Titterington, D.M. (1975). "Optimal Design: Some Geometrical Aspects of D optimality". Biometrika 62, pp. 313-319.

Williams, J.D., Woodall, W.H., and Birch, J.B. (2005). "Phase I Analysis of Nonlinear Product and Process Quality Profiles". submitted for publication.

Williams, J.D., Woodall, W.H., Birch, J.B., and Sullivan, J.H. (2005). "On the Distribution of the Hotellings' $T^{2}$ Statistic Based on the Successive Differences Covariance Matrix Estimator". submitted for publication.

Wisnowski, J.W., Simpson, J.R., and Montgomery, D.C. (2002). "A Performance Study for Multivariate Location and Shape Estimators". Quality and Reliability Engineering International 18, pp. 117-129.

Woodruff, D.L. and Rocke, D.M. (1993). "Heuristic Search Algorithms for the Minimum Volume Ellipsoid". Journal of Computational and Graphical Statistics 2, pp. 69-95.

Woodruff, D.L. and Rocke, D.M. (1994). "Computable Robust Estimation of Multivariate Location and Shape in High Dimension Using Compound Generators". Journal of the American Statistical Association 89, pp. 888-896.

Vargas, J.A. (2003). "Robust Estimation in Multivariate Control Charts for Individual Observations". Journal of Quality Technology 35, pp. 367-376. 